\title{
CONVERGENCE ANALYSIS OF TWO FINITE ELEMENT METHODS FOR THE MODIFIED MAXWELL'S STEKLOV EIGENVALUE PROBLEM
}

\author{
Bo GonG*(1)
}

\begin{abstract}
The modified Maxwell's Steklov eigenvalue problem is a new problem arising from the study of inverse electromagnetic scattering problems. In this paper, we investigate two finite element methods for this problem and perform the convergence analysis. Moreover, the monotonic convergence of the discrete eigenvalues computed by one of the methods is analyzed.
\end{abstract}

Mathematics Subject Classification. 65N25, 65N30.

Received May 21, 2021. Accepted January 3, 2022.

\section{INTRODUCTION}

The Steklov eigenvalue problem is governed by the elliptic equation with the eigen-parameter in the boundary condition. It has many applications in physics, e.g., surface waves [4] and stability of mechanical oscillators immersed in a viscous fluid [13]. Various numerical methods for the Steklov eigenvalue problem have been developed and analyzed [1,6,19,22,24,26]. Recently, a new application was considered in [10] using the Steklov eigenvalues as a target signature in nondestructive testing (see e.g., $[12,17]$ for different choices of target signatures). The associated non-selfadjoint Steklov eigenvalue problem for inhomogeneous absorbing media has drawn significant attention in the numerical analysis community [20, 25, 27].

Most earlier papers on Steklov eigenvalues focused on the Laplace or the Helmholtz equation. For the Maxwell's equation, the so-called modified Steklov eigenvalues was studied in [11] for an electromagnetic inverse scattering problem. In the same paper a finite element method was proposed for computing the eigenvalues. The term "modified" refers to the insertion of a boundary-to-boundary operator $\mathcal{S}$ into the standard Steklov eigenvalue problem. Through this modification the authors showed the compactness of the corresponding solution operator and the existence of the eigenvalues. For numerical analysis about this eigenvalue problem, to our knowledge, there exist only two papers $[15,16]$. Halla [16] provided a general framework considering the original and the modified Maxwell's Steklov eigenvalue problem, which guaranteed that the Galerkin approximation is convergent as long as certain commuting projection operator exists. While in [15], a specific finite element method was considered and a convergence order of the corresponding discrete eigenvalues were obtained. However, neither of these two results cover the method proposed in [11].

The difference between the two finite element methods, the one used in [11] and the one considered in [15], is how $\mathcal{S}$ is discretized. The boundary-to-boundary operator $\mathcal{S}$ projects vectors into the surface-divergence-free

Keywords and phrases. Steklov eigenvalues, Maxwell's equation, finite element method.

Faculty of Science, Beijing University of Technology, Beijing 100124, P.R. China.

*Corresponding author: gongbo@bjut.edu.cn 
space. It has two equivalent representations on the continuous level, but the corresponding finite element discretizations differ substantially. We denote by $\mathcal{S}_{h}$ the discretization used in [11], and by $\widetilde{\mathcal{S}}_{h}$ the one in [15]. What is crucial of $\widetilde{\mathcal{S}}_{h}$ is that it maintains the property of $\mathcal{S}$ to map vectors into the surface-divergence-free space. In contrast, $\mathcal{S}_{h}$ does not, i.e., it has a range not surface-divergence-free. Despite of this difference between $\widetilde{\mathcal{S}}_{h}$ and $\mathcal{S}_{h}$, they display similar numerical behaviour (see [15]). Both the eigenvalues $\lambda_{h}$ and $\widetilde{\lambda}_{h}$ computed by the method using $\mathcal{S}_{h}$ and $\widetilde{\mathcal{S}}_{h}$ respectively, converge to the exact values. Moreover, $\lambda_{h}$ seems to be more accurate than $\widetilde{\lambda}_{h}$. This motivates us to analyze the finite element method that uses $\mathcal{S}_{h}$, which is the main goal of the current paper.

As mentioned above, the major difficulty in the analysis lies in the fact that the range of $\mathcal{S}_{h}$ is not surfacedivergence-free. To this end, we define a solution operator slightly different than that from [11] so that its domain and range are the $L^{2}$ space other than the surface-divergence-free space. Both finite element methods (with $\widetilde{\mathcal{S}}_{h}$ and $\mathcal{S}_{h}$ ) under this $L^{2}$-to- $L^{2}$ framework are analyzed. The main tools used here are the Helmholtz decomposition and the Babuška-Osborn theory for eigenvalue problems [3] (see $[5,23]$ for some recent developments). In addition, we prove under some conditions the monotonic convergence of $\widetilde{\lambda}_{h}$ and an inequality between $\lambda_{h}$ and $\widetilde{\lambda}_{h}$.

The rest of the paper is arranged as follows. In Section 2, we introduce the notations and present some useful estimations and identities. Sections 3 and 4 contain the error analysis for the finite element methods using $\widetilde{\mathcal{S}}_{h}$ and $\mathcal{S}_{h}$, respectively. Convergence in norm of the discrete solution operators and the convergence order of the associated eigenvalues are obtained. In Section 5, we prove some other properties for the discrete eigenvalues.

\section{Preliminary}

Let $\Omega \subset \mathbb{R}^{3}$ be a simply connected bounded Lipschitz polyhedron with a connected boundary $\Gamma$. Let $\boldsymbol{\nu}$ be the unit outward normal to $\Gamma$. Denote by $H^{s}(\Omega)$ and $H^{t}(\Gamma)$ the standard Sobolev spaces for $s \in \mathbb{R}$ and $t \in[-1,1]$, respectively. Define

$$
\begin{aligned}
\boldsymbol{H}^{s}(\Omega) & :=\left(H^{s}(\Omega)\right)^{3}, \boldsymbol{L}^{2}(\Omega):=\left(L^{2}(\Omega)\right)^{3}, \boldsymbol{L}^{2}(\Gamma):=\left(L^{2}(\Gamma)\right)^{3}, \\
\boldsymbol{H}(\operatorname{curl} ; \Omega) & :=\left\{\boldsymbol{u} \in \boldsymbol{L}^{2}(\Omega) \mid \operatorname{curl} \boldsymbol{u} \in \boldsymbol{L}^{2}(\Omega)\right\}, \\
\boldsymbol{H}(\operatorname{div} ; \Omega) & :=\left\{\boldsymbol{u} \in \boldsymbol{L}^{2}(\Omega) \mid \operatorname{div} \boldsymbol{u} \in L^{2}(\Omega)\right\}, \\
\boldsymbol{H}_{0}(\operatorname{div} ; \Omega) & :=\{\boldsymbol{u} \in \boldsymbol{H}(\operatorname{div} ; \Omega) \mid \boldsymbol{\nu} \cdot \boldsymbol{u}=0 \text { a.e. on } \Gamma\}, \\
\boldsymbol{L}_{t}^{2}(\Gamma) & :=\left\{\boldsymbol{\mu} \in \boldsymbol{L}^{2}(\Gamma) \mid \boldsymbol{\nu} \cdot \boldsymbol{\mu}=0 \text { a.e. on } \Gamma\right\} .
\end{aligned}
$$

We denote the norm of $\boldsymbol{H}^{s}(\Omega), \boldsymbol{L}_{t}^{2}(\Gamma)$ and $H^{t}(\Gamma)$ as $\|\cdot\|_{s, \Omega},\|\cdot\|_{0, \Gamma}$ and $\|\cdot\|_{t, \Gamma}$, respectively. The norm of $\boldsymbol{H}(\mathbf{c u r l} ; \Omega)$ is $\|\cdot\|_{\operatorname{curl}, \Omega}$ with $\|\boldsymbol{u}\|_{\operatorname{curl}, \Omega}^{2}:=\|\boldsymbol{u}\|_{0, \Omega}^{2}+\|\operatorname{curl} \boldsymbol{u}\|_{0, \Omega}^{2}$.

Denote by $\Gamma_{j}, j=1, \ldots, J$, the boundary faces of $\Omega$. For $\psi \in L^{2}(\Gamma)$, let $\psi_{j}=\left.\psi\right|_{\Gamma_{j}}$. The spaces $H^{1+t}(\Gamma)$ and $\boldsymbol{H}_{-}^{t}(\Gamma)$ for $t>0$ are defined as [8]

$$
H^{1+t}(\Gamma)=\left\{\psi \in H^{1}(\Gamma) \mid \psi_{j} \in H^{1+t}\left(\Gamma_{j}\right)\right\} \quad \text { and } \quad \boldsymbol{H}_{-}^{t}(\Gamma)=\left\{\boldsymbol{\phi} \in \boldsymbol{L}_{t}^{2}(\Gamma) \mid \boldsymbol{\phi}_{j} \in H^{t}\left(\Gamma_{j}\right)^{2}\right\},
$$

with $\|\psi\|_{1+t, \Gamma}^{2}:=\|\psi\|_{1, \Gamma}^{2}+\sum_{j=1}^{J}\left\|\psi_{j}\right\|_{1+t, \Gamma_{j}}^{2}$ and $\|\phi\|_{t,-, \Gamma}^{2}:=\sum_{j=1}^{J}\left\|\phi_{j}\right\|_{H^{t}\left(\Gamma_{j}\right)^{2}}^{2}$.

Let

$$
\gamma_{t}:\left(C^{\infty}(\bar{\Omega})\right)^{3} \rightarrow \boldsymbol{L}_{t}^{2}(\Gamma) \quad \text { and } \quad \gamma_{T}:\left(C^{\infty}(\bar{\Omega})\right)^{3} \rightarrow \boldsymbol{L}_{t}^{2}(\Gamma)
$$

be the trace operators that maps $\boldsymbol{v}$ to $\boldsymbol{\nu} \times\left.\boldsymbol{v}\right|_{\Gamma}$ and $\left(\boldsymbol{\nu} \times\left.\boldsymbol{v}\right|_{\Gamma}\right) \times \boldsymbol{\nu}$, respectively. The operators $\gamma_{t}$ and $\gamma_{T}$ can be continuously extended to $\boldsymbol{H}(\mathbf{c u r l} ; \Omega)$. Denote by $\boldsymbol{v}_{T}=\gamma_{T} \boldsymbol{v}$ the tangential component of $\boldsymbol{v}$ on the boundary. 
Let $\nabla_{\Gamma}$ and curl $_{\Gamma}$ denote, respectively, the surface gradient and surface vector curl, which can be defined on $H^{1 / 2}(\Gamma)$. The surface divergence and surface scalar curl, denoted by $\operatorname{div}_{\Gamma}$ and $\operatorname{curl}_{\Gamma}$, are respectively the duals of $-\nabla_{\Gamma}$ and $\operatorname{curl}_{\Gamma}$, i.e.,

$$
\left\langle\phi, \nabla_{\Gamma} \psi\right\rangle=-\left\langle\operatorname{div}_{\Gamma} \phi, \psi\right\rangle, \quad\left\langle\phi, \operatorname{curl}_{\Gamma} \psi\right\rangle=\left\langle\operatorname{curl}_{\Gamma} \phi, \psi\right\rangle .
$$

Define the surface-divergence-free space as

$$
\boldsymbol{H}\left(\operatorname{div}_{\Gamma}^{0} ; \Gamma\right):=\left\{\boldsymbol{\mu} \in \boldsymbol{L}_{t}^{2}(\Gamma) \mid \operatorname{div}_{\Gamma} \boldsymbol{\mu}=0\right\} .
$$

For more details on these operators and spaces, we refer the readers to $[7,9]$.

In this paper, we consider the modified Maxwell's Steklov eigenvalue problem of finding $(\lambda, \boldsymbol{u}) \in \mathbb{R} \times$ $\boldsymbol{H}(\operatorname{curl} ; \Omega)$ such that

$$
(\operatorname{curl} \boldsymbol{u}, \operatorname{curl} \boldsymbol{v})-\kappa^{2}\left(\epsilon_{r} \boldsymbol{u}, \boldsymbol{v}\right)=-\lambda\left\langle\mathcal{S} \boldsymbol{u}_{T}, \boldsymbol{v}_{T}\right\rangle, \quad \forall \boldsymbol{v} \in \boldsymbol{H}(\operatorname{curl} ; \Omega) .
$$

Here $\kappa$ is the wavenumber which is real and positive and $\epsilon_{r}$ is the relative permittivity. Assume that the media is isotropic and dielectric, i.e., $\epsilon_{r}$ is a real scalar function. In addition, we require that $\epsilon_{r}$ is smooth, bounded and away from zero. More precisely, there exist constants $\alpha>0$ and $\beta>0$ such that $\epsilon_{r} \in H^{1}(\Omega)$ and $\alpha \leqslant \epsilon_{r} \leqslant \beta$. For $\boldsymbol{u}, \boldsymbol{v} \in \boldsymbol{L}^{2}(\Omega)$ and $\boldsymbol{f}, \boldsymbol{g} \in \boldsymbol{L}_{t}^{2}(\Gamma)$, define

$$
(\boldsymbol{u}, \boldsymbol{v})=\int_{\Omega} \boldsymbol{u}(\boldsymbol{x}) \cdot \boldsymbol{v}(\boldsymbol{x}) \mathrm{d} V(\boldsymbol{x}) \quad \text { and } \quad\langle\boldsymbol{f}, \boldsymbol{g}\rangle=\int_{\Gamma} \boldsymbol{f}(\boldsymbol{x}) \cdot \boldsymbol{g}(\boldsymbol{x}) \mathrm{d} A(\boldsymbol{x}) .
$$

We also use $\langle\cdot, \cdot\rangle$ to denote certain duality of spaces on the boundary. There are two equivalent ways to define the surface-divergence-free projection operator $\mathcal{S}$. One is $\mathcal{S} \boldsymbol{\mu}=\operatorname{curl}_{\Gamma} q$ with $q \in H^{1}(\Gamma) / \mathbb{R}$ satisfying

$$
\left\langle\operatorname{curl}_{\Gamma} q, \operatorname{curl}_{\Gamma} \psi\right\rangle=\left\langle\boldsymbol{\mu}, \operatorname{curl}_{\Gamma} \psi\right\rangle, \quad \forall \psi \in H^{1}(\Gamma) .
$$

The above definition can be applied on, say, $\boldsymbol{\mu} \in \gamma_{T} \boldsymbol{H}(\mathbf{c u r l} ; \Omega)$ or $\boldsymbol{\mu} \in \boldsymbol{L}_{t}^{2}(\Gamma)$. The other is $\mathcal{S} \boldsymbol{\mu}=\boldsymbol{\mu}+\nabla_{\Gamma} p$ with $p \in H^{1}(\Gamma) / \mathbb{R}$ being the solution of

$$
\left\langle\nabla_{\Gamma} p, \nabla_{\Gamma} \psi\right\rangle=-\left\langle\boldsymbol{\mu}, \nabla_{\Gamma} \psi\right\rangle, \quad \forall \psi \in H^{1}(\Gamma),
$$

which can be applied on, say, $\boldsymbol{\mu} \in \gamma_{t} \boldsymbol{H}(\mathbf{c u r l} ; \Omega)$ or $\boldsymbol{\mu} \in \boldsymbol{L}_{t}^{2}(\Gamma)$.

Let $\tau_{h}$ be a regular tetrahedral mesh for polyhedron $\Omega$ with size $h$. The faces of $\tau_{h}$ on $\Gamma$ induce a triangular mesh for $\Gamma$. We use the notations in Chapter 5 of [21] to denote by $W_{h} \subset \boldsymbol{H}$ (div, $\Omega$ ) the divergence-conforming finite element space of degree $k$, by $V_{h} \subset \boldsymbol{H}(\mathbf{c u r l} ; \Omega)$ the curl-conforming finite element space of degree $k$, and by $U_{h} \subset H^{1}(\Omega)$ the Lagrange element space of degree $k$. In addition, denote by $U_{h}^{\Gamma} \subset H^{1}(\Gamma)$ the Lagrange element space of degree $k$ on the boundary. We shall mainly discuss the case when $k=1$.

Denote by $\pi_{h}^{1}: \boldsymbol{H}(\operatorname{curl} ; \Omega) \supset \mathcal{V} \rightarrow V_{h}$ and $\pi_{h}^{2}: \boldsymbol{H}(\operatorname{div} ; \Omega) \supset \mathcal{W} \rightarrow W_{h}$ the interpolation operators. Here $\mathcal{V}$ and $\mathcal{W}$ are suitable subspaces such that the interpolations are well-defined and bounded (see e.g., [21], Lem. 5.38). The finite element spaces $W_{h}, V_{h}$ and $U_{h}$ satisfy the de Rham complex (see e.g., [21], (5.59)), which implies

$$
\operatorname{curl} V_{h} \subset W_{h}, \quad \nabla U_{h} \subset V_{h}, \quad \text { and } \quad \operatorname{curl} \pi_{h}^{1} \boldsymbol{v}=\pi_{h}^{2} \operatorname{curl} \boldsymbol{v} \quad \text { for } \boldsymbol{v} \in \mathcal{V} .
$$

Moreover, the kernel of curl in $V_{h}$ is $\nabla U_{h}$ (see e.g., [2]).

Based on the definitions of $\mathcal{S}$ by (2.2) and (2.3), the two finite element approximations $\widetilde{\mathcal{S}}_{h}$ and $\mathcal{S}_{h}$ are defined as follows. $\widetilde{\mathcal{S}}_{h}: \boldsymbol{L}_{t}^{2}(\Gamma) \rightarrow \boldsymbol{L}_{t}^{2}(\Gamma)$ is such that $\widetilde{\mathcal{S}}_{h} \boldsymbol{\mu}=\operatorname{curl}_{\Gamma} q_{h}$ with $q_{h} \in U_{h}^{\Gamma} / \mathbb{R}$ being the solution of

$$
\left\langle\operatorname{curl}_{\Gamma} q_{h}, \operatorname{curl}_{\Gamma} \psi_{h}\right\rangle=\left\langle\boldsymbol{\mu}, \operatorname{curl}_{\Gamma} \psi_{h}\right\rangle, \quad \forall \psi_{h} \in U_{h}^{\Gamma} .
$$

And $\mathcal{S}_{h}: \boldsymbol{L}_{t}^{2}(\Gamma) \rightarrow \boldsymbol{L}_{t}^{2}(\Gamma)$ is such that $\mathcal{S}_{h} \boldsymbol{\mu}=\boldsymbol{\mu}+\nabla_{\Gamma} p_{h}$ with $p_{h} \in U_{h}^{\Gamma} / \mathbb{R}$ being the solution of

$$
\left\langle\nabla_{\Gamma} p_{h}, \nabla_{\Gamma} \psi_{h}\right\rangle=-\left\langle\boldsymbol{\mu}, \nabla_{\Gamma} \psi_{h}\right\rangle, \quad \forall \psi_{h} \in U_{h}^{\Gamma} .
$$


Note that $\widetilde{\mathcal{S}}_{h}$ and $\mathcal{S}_{h}$ can be defined on larger spaces than $\boldsymbol{L}_{t}^{2}(\Gamma)$.

In the rest of this section, we collect some results that will be used later. All except the last two of these lemmas can be found in [15] and references therein. Let $Z \subset \boldsymbol{H}(\operatorname{curl} ; \Omega)$ and $Z_{h} \in V_{h}$ be such that

$$
\begin{aligned}
Z & =\left\{\boldsymbol{u} \in \boldsymbol{H}(\mathbf{c u r l} ; \Omega) \mid\left(\epsilon_{r} \boldsymbol{u}, \nabla p\right)=0, \forall p \in H^{1}(\Omega)\right\} \\
\text { and } \quad Z_{h} & =\left\{\boldsymbol{u}_{h} \in V_{h} \mid\left(\epsilon_{r} \boldsymbol{u}_{h}, \nabla p_{h}\right)=0, \forall p_{h} \in U_{h}\right\} .
\end{aligned}
$$

Lemma 2.1 (see e.g., [15], Lem. 2.1 and Sect. 3.1). The spaces $\boldsymbol{H}(\mathbf{c u r l} ; \Omega)$ and $V_{h}$ can be decomposed, respectively, as

$$
\boldsymbol{H}(\operatorname{curl} ; \Omega)=Z \oplus \nabla\left(H^{1}(\Omega) / \mathbb{R}\right) \quad \text { and } \quad V_{h}=Z_{h} \oplus \nabla\left(U_{h} / \mathbb{R}\right) .
$$

Definition 2.2. Denote by $\mathcal{P}: \boldsymbol{H}(\operatorname{curl} ; \Omega) \rightarrow Z$ and $\mathcal{P}_{h}: V_{h} \rightarrow Z_{h}$, respectively, the projection operator according to Lemma 2.1 .

Lemma 2.3 (see e.g., [15], Lem. 2.2). For $0<s<s_{\Omega}$ with some parameter $s_{\Omega}>0$ dependent on the geometry of $\Omega$, it holds that

$$
Z \subset \boldsymbol{H}(\operatorname{curl} ; \Omega) \cap \boldsymbol{H}_{0}(\operatorname{div} ; \Omega) \subset \boldsymbol{H}^{1 / 2+s}(\Omega) \quad \text { and } \quad \gamma_{T} Z \subset \boldsymbol{H}_{-}^{s}(\Gamma)
$$

and for $\boldsymbol{u} \in Z$

$$
\|\boldsymbol{u}\|_{1 / 2+s, \Omega} \leqslant C\|\boldsymbol{u}\|_{\operatorname{curl}, \Omega} \quad \text { and } \quad\left\|\boldsymbol{u}_{T}\right\|_{\boldsymbol{H}_{-}^{s}(\Gamma)} \leqslant C\|\boldsymbol{u}\|_{\operatorname{curl}, \Omega} .
$$

From now on we use $s$ to represent some positive number which is less than $s_{\Omega}$.

Lemma 2.4 (see e.g., the proof of Lem. 3.4 from [15]). For $\boldsymbol{z}_{h} \in Z_{h}$, it holds

$$
\left\|(I-\mathcal{P}) \boldsymbol{z}_{h}\right\|_{\mathbf{c u r l}, \Omega} \leqslant C h^{1 / 2+s}\left\|\boldsymbol{z}_{h}\right\|_{\mathbf{c u r l}, \Omega} .
$$

Next, we consider the continuous and discrete source problems, as well as the associated operators. Denote by $a(\cdot, \cdot)$ and $a_{+}(\cdot, \cdot)$ the sesquilinear forms on $\boldsymbol{H}(\operatorname{curl} ; \Omega) \times \boldsymbol{H}(\mathbf{c u r l} ; \Omega)$ such that

$$
\begin{aligned}
a(\boldsymbol{u}, \boldsymbol{v}) & =(\operatorname{curl} \boldsymbol{u}, \operatorname{curl} \boldsymbol{v})-\kappa^{2}\left(\epsilon_{r} \boldsymbol{u}, \boldsymbol{v}\right) \\
a_{+}(\boldsymbol{u}, \boldsymbol{v}) & =(\operatorname{curl} \boldsymbol{u}, \operatorname{curl} \boldsymbol{v})+\left(\epsilon_{r} \boldsymbol{u}, \boldsymbol{v}\right)
\end{aligned}
$$

Formally, let $\mathcal{L}: \boldsymbol{H}\left(\operatorname{div}_{\Gamma}^{0} ; \Gamma\right) \rightarrow \boldsymbol{H}(\operatorname{curl} ; \Omega), \mathcal{L}_{h}: \boldsymbol{H}\left(\operatorname{div}_{\Gamma}^{0} ; \Gamma\right) \rightarrow V_{h}, \mathcal{L}^{+}: \boldsymbol{H}\left(\operatorname{div}_{\Gamma}^{0} ; \Gamma\right) \rightarrow \boldsymbol{H}(\operatorname{curl} ; \Omega), \mathcal{L}_{h}^{+}:$ $\boldsymbol{H}\left(\operatorname{div}_{\Gamma}^{0} ; \Gamma\right) \rightarrow V_{h}, \mathcal{K}: \boldsymbol{L}^{2}(\Omega) \rightarrow Z$ and $\mathcal{K}_{h}: \boldsymbol{L}^{2}(\Omega) \rightarrow Z_{h}$ be the operators such that

$$
\begin{aligned}
a(\mathcal{L} \boldsymbol{f}, \boldsymbol{v}) & =\left\langle\boldsymbol{f}, \boldsymbol{v}_{T}\right\rangle, & & \forall \boldsymbol{v} \in \boldsymbol{H}(\mathbf{c u r l} ; \Omega), \\
a\left(\mathcal{L}_{h} \boldsymbol{f}, \boldsymbol{v}_{h}\right) & =\left\langle\boldsymbol{f}, \boldsymbol{v}_{h, T}\right\rangle, & & \forall \boldsymbol{v}_{h} \in V_{h}, \\
a_{+}\left(\mathcal{L}^{+} \boldsymbol{f}, \boldsymbol{v}\right) & =\left\langle\boldsymbol{f}, \boldsymbol{v}_{T}\right\rangle, & & \forall \boldsymbol{v} \in \boldsymbol{H}(\mathbf{c u r l} ; \Omega), \\
a_{+}\left(\mathcal{L}_{h}^{+} \boldsymbol{f}, \boldsymbol{v}_{h}\right) & =\left\langle\boldsymbol{f}, \boldsymbol{v}_{h, T}\right\rangle, & & \forall \boldsymbol{v}_{h} \in V_{h}, \\
a_{+}(\mathcal{K} \boldsymbol{w}, \boldsymbol{v}) & =-\left(\kappa^{2}+1\right)\left(\epsilon_{r} \boldsymbol{w}, \boldsymbol{v}\right), & & \forall \boldsymbol{v} \in Z, \\
a_{+}\left(\mathcal{K}_{h} \boldsymbol{w}, \boldsymbol{v}_{h}\right) & =-\left(\kappa^{2}+1\right)\left(\epsilon_{r} \boldsymbol{w}, \boldsymbol{v}_{h}\right), & & \forall \boldsymbol{v}_{h} \in Z_{h},
\end{aligned}
$$

for $\boldsymbol{f} \in \boldsymbol{H}\left(\operatorname{div}_{\Gamma}^{0} ; \Gamma\right)$ and $\boldsymbol{w} \in \boldsymbol{L}^{2}(\Omega)$. Note that the domain of $\mathcal{L}, \mathcal{L}_{h}, \mathcal{L}^{+}$and $\mathcal{L}_{h}^{+}$are set to be $\boldsymbol{H}\left(\operatorname{div}_{\Gamma}^{0} ; \Gamma\right)$, a strict subset of $\boldsymbol{L}_{t}^{2}(\Gamma)$. The source problems (2.9)-(2.12) are clearly well-posed. For the well-posedness of (2.7) and (2.8), we assume the uniqueness, that is, we assume that $\kappa^{2}$ is not a Neumann eigenvalue defined below. 
Definition 2.5. We call $\kappa^{2}$ a Neumann eigenvalue of the Maxwell's equation if there exists a non-trivial function $\boldsymbol{u}$ such that

$$
\begin{aligned}
\operatorname{curl} \operatorname{curl} \boldsymbol{u}-\kappa^{2} \epsilon_{r} \boldsymbol{u} & =\mathbf{0}, & & \text { in } \Omega, \\
\boldsymbol{\nu} \times \operatorname{curl} \boldsymbol{u} & =\mathbf{0}, & & \text { on } \Gamma .
\end{aligned}
$$

Definition 2.6. Let $\Lambda=\left\{h_{n}\right\}_{n=1}^{\infty}$ be such that $h_{n} \rightarrow 0$ as $n \rightarrow \infty$. Let $X$ denote a general Hilbert space. A set $\left\{\mathcal{F}_{h} \mid X \rightarrow X, h \in \Lambda\right\}$ of bounded linear operators is called collectively compact if, for each bounded set $U \subset X$, the image set $\left\{\mathcal{F}_{h} u \mid \forall u \in U, \forall h \in \Lambda\right\}$ is relatively compact.

It can be shown that $\mathcal{K}$ is compact and $\left\{\mathcal{K}_{h}\right\}_{h}$ is collectively compact as operators from $\boldsymbol{L}^{2}(\Omega)$ to itself (see e.g., [15], Sects. 2 and 3). As a consequence the source problems (2.7) and (2.8) are well-posed. We gather in the following lemma the well-posedness of the problems (2.7)-(2.10).

Lemma 2.7 (see e.g., [21], Thm. 2.51, [15], Sects. 2 and 3). For $h$ small enough, the operators $(I+\mathcal{K})^{-1}$ and $\left(I+\mathcal{K}_{h}\right)^{-1}$ exist and are (uniformly in $h$ ) bounded from $\boldsymbol{L}^{2}(\Omega)$ to $\boldsymbol{L}^{2}(\Omega)$. Moreover, $\mathcal{L}$ and $\mathcal{L}_{h}$ have the following representations,

$$
\mathcal{L}=(I+\mathcal{K})^{-1} \mathcal{L}^{+} \quad \text { and } \quad \mathcal{L}_{h}=\left(I+\mathcal{K}_{h}\right)^{-1} \mathcal{L}_{h}^{+} .
$$

$\mathcal{L}^{+}, \mathcal{L}_{h}^{+}, \mathcal{L}$ and $\mathcal{L}_{h}$ are (uniformly) bounded.

It is easily seen that $\operatorname{ran}(\mathcal{L}) \subset Z$ and $\operatorname{ran}\left(\mathcal{L}_{h}\right) \subset Z_{h}$. One has the following error estimate.

Lemma 2.8 ([15], Thm. 3.7). $\left\|\mathcal{L}-\mathcal{L}_{h}\right\| \leqslant C h^{1 / 2}$.

To obtain regularity of the solution of (2.2) and (2.3), we shall apply the following result.

Lemma 2.9 ([8], Thm. 8). Given $g \in H^{r}(\Gamma)$ with $r>-1$, the solution $p \in H^{1}(\Gamma) / \mathbb{R}$ of

$$
-\Delta_{\Gamma} p=g
$$

admits $p \in H^{1+t}(\Gamma) / \mathbb{R}$ with $t<\min \left\{s_{\Gamma}, 1+r\right\}$, where $s_{\Gamma}>0$ is some parameter depending on the shape of $\Gamma$.

A direct use of the definitions of $\mathcal{S}, \widetilde{\mathcal{S}}_{h}$ and $\mathcal{S}_{h}$ gives one the following.

Lemma 2.10. The operators $\mathcal{S}, \widetilde{\mathcal{S}}_{h}$ and $\mathcal{S}_{h}$ are linear and bounded from $\boldsymbol{L}_{t}^{2}(\Gamma)$ to $\boldsymbol{L}_{t}^{2}(\Gamma)$. Moreover, they are orthogonal projection operators under the $\boldsymbol{L}_{t}^{2}(\Gamma)$ inner product, and given $\boldsymbol{f}, \boldsymbol{g} \in \boldsymbol{L}_{t}^{2}(\Gamma)$, it holds that

$$
\begin{aligned}
\langle\mathcal{S} \boldsymbol{f}, \boldsymbol{g}\rangle & =\langle\mathcal{S} \boldsymbol{f}, \mathcal{S} \boldsymbol{g}\rangle=\langle\boldsymbol{f}, \mathcal{S} \boldsymbol{g}\rangle=\left\langle\mathcal{S}_{h} \boldsymbol{f}, \mathcal{S} \boldsymbol{g}\right\rangle, \\
\left\langle\widetilde{\mathcal{S}}_{h} \boldsymbol{f}, \boldsymbol{g}\right\rangle & =\left\langle\widetilde{\mathcal{S}}_{h} \boldsymbol{f}, \widetilde{\mathcal{S}}_{h} \boldsymbol{g}\right\rangle=\left\langle\boldsymbol{f}, \widetilde{\mathcal{S}}_{h} \boldsymbol{g}\right\rangle=\left\langle\mathcal{S} \boldsymbol{f}, \widetilde{\mathcal{S}}_{h} \boldsymbol{g}\right\rangle=\left\langle\mathcal{S}_{h} \boldsymbol{f}, \widetilde{\mathcal{S}}_{h} \boldsymbol{g}\right\rangle, \\
\left\langle\mathcal{S}_{h} \boldsymbol{f}, \boldsymbol{g}\right\rangle & =\left\langle\mathcal{S}_{h} \boldsymbol{f}, \mathcal{S}_{h} \boldsymbol{g}\right\rangle=\left\langle\boldsymbol{f}, \mathcal{S}_{h} \boldsymbol{g}\right\rangle,
\end{aligned}
$$

which implies that on $\boldsymbol{L}_{t}^{2}(\Gamma)$ we have

$$
\mathcal{S} \mathcal{S}_{h}=\mathcal{S}_{h} \mathcal{S}=\mathcal{S}, \quad \widetilde{\mathcal{S}}_{h} \mathcal{S}=\mathcal{S} \widetilde{\mathcal{S}}_{h}=\widetilde{\mathcal{S}}_{h}, \quad \widetilde{\mathcal{S}}_{h} \mathcal{S}_{h}=\mathcal{S}_{h} \widetilde{\mathcal{S}}_{h}=\widetilde{\mathcal{S}}_{h} .
$$

Proof. Given $\boldsymbol{f}, \boldsymbol{g} \in \boldsymbol{L}_{t}^{2}(\Gamma)$, we have by definition $\phi_{h} \in U_{h}^{\Gamma}, \phi \in H^{1}(\Gamma)$ and $\psi \in H^{1}(\Gamma)$ such that $\mathcal{S}_{h} \boldsymbol{f}=$ $\boldsymbol{f}+\nabla_{\Gamma} \phi_{h}, \mathcal{S} \boldsymbol{f}=\operatorname{curl}_{\Gamma} \phi$ and $\mathcal{S} \boldsymbol{g}=\operatorname{curl}_{\Gamma} \psi$. Then by the definition of $\mathcal{S} \boldsymbol{f}$ and $\mathcal{S} \boldsymbol{g}$ we have the following

$$
\langle(I-\mathcal{S}) \boldsymbol{f}, \mathcal{S} \boldsymbol{g}\rangle=\left\langle(I-\mathcal{S}) \boldsymbol{f}, \operatorname{curl}_{\Gamma} \psi\right\rangle=0, \quad\left\langle\left(I-\mathcal{S}_{h}\right) \boldsymbol{f}, \mathcal{S} \boldsymbol{g}\right\rangle=\left\langle-\nabla_{\Gamma} \phi_{h}, \operatorname{curl}_{\Gamma} \psi\right\rangle=0 .
$$

Hence we have the first line of equalities verified, by which we can show that

$$
\left\langle\mathcal{S} \mathcal{S}_{h} \boldsymbol{f}, \boldsymbol{g}\right\rangle=\left\langle\mathcal{S}_{h} \boldsymbol{f}, \mathcal{S} \boldsymbol{g}\right\rangle=\langle\boldsymbol{f}, \mathcal{S} \boldsymbol{g}\rangle=\langle\mathcal{S} \boldsymbol{f}, \boldsymbol{g}\rangle,
$$

which means $\mathcal{S S}_{h}=\mathcal{S}$ on $\boldsymbol{L}_{t}^{2}(\Gamma)$. The rest can be proved similarly. 
Note that $\mathcal{S}$ can be defined on $\gamma_{T} \boldsymbol{H}(\mathbf{c u r l} ; \Omega)$, and $\mathcal{S}$ is still self-adjoint on $\gamma_{T} \boldsymbol{H}(\operatorname{curl} ; \Omega)$ (see [11], Lem. 3.1).

In [11] the solution operator of $(2.1)$ is defined from $\boldsymbol{H}\left(\operatorname{div}_{\Gamma}^{0} ; \Gamma\right)$ to itself by $\mathcal{S} \gamma_{T} \mathcal{L}$, which is inconvenient for the current paper. Instead, we define for (2.1) another solution operator $\mathcal{T}$ such that

$$
\mathcal{T}: \boldsymbol{L}_{t}^{2}(\Gamma) \rightarrow \boldsymbol{L}_{t}^{2}(\Gamma), \quad \mathcal{T}=\mathcal{S} \gamma_{T} \mathcal{L} \mathcal{S}
$$

We show in the following that $\mathcal{T}$ is compact and indeed represents the spectrum of (2.1).

Lemma 2.11. $\mathcal{T}$ is compact and self-adjoint. There is a bijection between the eigenpairs of $\mathcal{T}$ and those of (2.1) for nonzero eigenvalues.

Proof. Given $\boldsymbol{f} \in \boldsymbol{L}_{t}^{2}(\Gamma), q \in H^{1}(\Gamma) / \mathbb{R}$ in $(2.2)$ is well-defined, thus $\mathcal{S} \boldsymbol{f} \in \boldsymbol{H}\left(\operatorname{div}_{\Gamma}^{0} ; \Gamma\right)$. Then due to the wellposedness of $\mathcal{L}$, it holds $\boldsymbol{u}:=\mathcal{L} \mathcal{S} \boldsymbol{f} \in Z$. Using the remark of [14], we have that $\operatorname{curl}_{\Gamma} \boldsymbol{u}_{T} \in L^{2}(\Gamma)$ and

$$
\left\|\operatorname{curl}_{\Gamma} \boldsymbol{u}_{T}\right\|_{0, \Gamma}=\|\boldsymbol{\nu} \cdot \operatorname{curl} \boldsymbol{u}\|_{0, \Gamma} \leqslant C\|\boldsymbol{u}\|_{\operatorname{curl}, \Omega}+C\|\mathcal{S} \boldsymbol{f}\|_{0, \Gamma} \leqslant C\|\mathcal{S} \boldsymbol{f}\|_{0, \Gamma} \leqslant C\|\boldsymbol{f}\|_{0, \Gamma} .
$$

Therefore, the regularity result of Lemma 2.9 indicates that for the solution $q \in H^{1}(\Gamma) / \mathbb{R}$ of

$$
\left\langle\operatorname{curl}_{\Gamma} q, \operatorname{curl}_{\Gamma} \psi\right\rangle=\left\langle\boldsymbol{u}_{T}, \operatorname{curl}_{\Gamma} \psi\right\rangle, \quad \forall \psi \in H^{1}(\Gamma),
$$

it holds that $q \in H^{1+t}(\Gamma) / \mathbb{R}$ with $t<\min \left\{s_{\Gamma}, 1\right\}$. Consequently,

$$
\mathcal{T} \boldsymbol{f}=\mathcal{S} \boldsymbol{u}_{T}=\operatorname{curl}_{\Gamma} q \in \boldsymbol{H}_{-}^{t}(\Gamma) \cap \boldsymbol{H}\left(\operatorname{div}_{\Gamma}^{0} ; \Gamma\right) .
$$

Since $\boldsymbol{H}_{-}^{t}(\Gamma) \cap \boldsymbol{H}\left(\operatorname{div}_{\Gamma}^{0} ; \Gamma\right)$ is compactly embedded in $\boldsymbol{L}_{t}^{2}(\Gamma), \mathcal{T}$ is well-defined and compact.

Given $\boldsymbol{f}, \boldsymbol{g} \in \boldsymbol{L}_{t}^{2}(\Gamma)$, we have

$$
\begin{aligned}
\langle\mathcal{T} \boldsymbol{f}, \boldsymbol{g}\rangle & =\left\langle\mathcal{S} \gamma_{T} \mathcal{L} \mathcal{S} \boldsymbol{f}, \boldsymbol{g}\right\rangle=\left\langle\gamma_{T} \mathcal{L} \mathcal{S} \boldsymbol{f}, \mathcal{S} \boldsymbol{g}\right\rangle=a(\mathcal{L} \mathcal{S} \boldsymbol{f}, \mathcal{L} \mathcal{S} \boldsymbol{g}) \\
& =\left\langle\mathcal{S} \boldsymbol{f}, \gamma_{T} \mathcal{L} \mathcal{S} \boldsymbol{g}\right\rangle=\left\langle\boldsymbol{f}, \mathcal{S} \gamma_{T} \mathcal{L} \mathcal{S} \boldsymbol{g}\right\rangle=\langle\boldsymbol{f}, \mathcal{T} \boldsymbol{g}\rangle
\end{aligned}
$$

Hence $\mathcal{T}$ is self-adjoint. Note that we have used the assumption that $\epsilon_{r}$ is real.

Given an eigenpair $(\lambda, \boldsymbol{u})$ of $(2.1), \lambda \neq 0$, we have $\mathcal{L}\left(\lambda \mathcal{S} \boldsymbol{u}_{T}\right)=\boldsymbol{u}$. Hence $\mathcal{S} \gamma_{T} \mathcal{L}\left(\lambda \mathcal{S} \boldsymbol{u}_{T}\right)=\mathcal{S} \boldsymbol{u}_{T}$, i.e., $\left(-1 / \lambda, \mathcal{S} \boldsymbol{u}_{T}\right)$ is an eigenpair of $\mathcal{T}$. On the other hand, given an eigenpair $(\mu, \boldsymbol{f})$ of $\mathcal{T}, \mu \neq 0$, then $\mathcal{T} \boldsymbol{f}=\mu \boldsymbol{f}$. Therefore $\mathcal{L}(1 / \mu) \mathcal{S} \gamma_{T} \mathcal{L} \boldsymbol{S} \boldsymbol{f}=\mathcal{L} \mathcal{S} \boldsymbol{f}$, i.e., $(-1 / \mu, \mathcal{L} \mathcal{S} \boldsymbol{f})$ is an eigenpair of $(2.1)$.

\section{Finite ELEMENT METHOD USING $\widetilde{\mathcal{S}}_{h}$}

For the completeness of the theory, also as a preparation for the next section, we first analyze the finite element method that uses $\widetilde{\mathcal{S}}_{h}$ as the discretization (see (2.5)). Instead of defining the corresponding solution operator $\widetilde{\mathcal{T}}_{h}$ (as is done in [15]) from $\boldsymbol{H}\left(\operatorname{div}_{\Gamma}^{0} ; \Gamma\right)$ to $\boldsymbol{H}\left(\operatorname{div}_{\Gamma}^{0} ; \Gamma\right)$, we shall define it from $\boldsymbol{L}_{t}^{2}(\Gamma)$ to $\boldsymbol{L}_{t}^{2}(\Gamma)$. One consequence is that, the operator $\widetilde{\mathcal{T}}_{h}$ thus defined is self-adjoint on $\boldsymbol{L}_{t}^{2}(\Gamma)$, while $\widetilde{\mathcal{T}}_{h}$ defined in [15] is not on $\boldsymbol{H}\left(\operatorname{div}_{\Gamma}^{0} ; \Gamma\right)$.

Consider the discrete eigenvalue problem of finding $\left(\widetilde{\lambda}_{h}, \boldsymbol{u}_{h}\right) \in \mathbb{R} \times V_{h}$ such that

$$
a\left(\boldsymbol{u}_{h}, \boldsymbol{v}_{h}\right)=-\widetilde{\lambda}_{h}\left\langle\widetilde{\mathcal{S}}_{h} \boldsymbol{u}_{h, T}, \boldsymbol{v}_{h, T}\right\rangle, \quad \forall \boldsymbol{v}_{h} \in V_{h} .
$$

Let the solution operator of (3.1) be defined as

$$
\widetilde{\mathcal{T}}_{h}: \boldsymbol{L}_{t}^{2}(\Gamma) \rightarrow \boldsymbol{L}_{t}^{2}(\Gamma), \quad \widetilde{\mathcal{T}}_{h}=\widetilde{\mathcal{S}}_{h} \gamma_{T} \mathcal{L}_{h} \widetilde{\mathcal{S}}_{h}
$$


Lemma 3.1. $\widetilde{\mathcal{T}}_{h}$ are uniformly bounded and self-adjoint. There is a bijection between the eigenpairs of (3.1) and those of $\widetilde{\mathcal{T}}_{h}$ for nonzero eigenvalues.

Proof. Since the range of $\widetilde{\mathcal{S}}_{h}$ is contained $\boldsymbol{H}\left(\operatorname{div}_{\Gamma}^{0} ; \Gamma\right), \mathcal{L}_{h} \widetilde{\mathcal{S}}_{h}$ are well-defined and uniformly bounded from $\boldsymbol{L}_{t}^{2}(\Gamma)$ to $\boldsymbol{L}_{t}^{2}(\Gamma)$. Hence $\widetilde{\mathcal{T}}_{h}$ are well-defined and uniformly bounded. Moreover, for $\boldsymbol{f} \in \boldsymbol{L}_{t}^{2}(\Gamma)$, noticing that $\gamma_{T}(I-\mathcal{P}) \mathcal{L}_{h} \widetilde{\mathcal{S}}_{h} \boldsymbol{f}=\nabla_{\Gamma} p$ for some $p \in H^{1 / 2}(\Gamma)$, we have $\widetilde{\mathcal{S}}_{h} \gamma_{T}(I-\mathcal{P}) \mathcal{L}_{h} \widetilde{\mathcal{S}}_{h} \boldsymbol{f}=0$. Therefore,

$$
\left\|\widetilde{\mathcal{T}}_{h}\right\|=\left\|\widetilde{\mathcal{S}}_{h} \gamma_{T} \mathcal{L}_{h} \widetilde{\mathcal{S}}_{h}\right\|=\left\|\widetilde{\mathcal{S}}_{h} \gamma_{T} \mathcal{P} \mathcal{L}_{h} \widetilde{\mathcal{S}}_{h}\right\| \leqslant C .
$$

That $\widetilde{\mathcal{T}}_{h}$ is self-adjoint and the correspondence of the eigenpairs between $\widetilde{\mathcal{T}}_{h}$ and (3.1) can be shown in the same way as in Lemma 2.11.

Next we prove the convergence of $\widetilde{\mathcal{T}}_{h}$ to $\mathcal{T}$.

Theorem 3.2. $\left\|\mathcal{T}-\widetilde{\mathcal{T}}_{h}\right\| \rightarrow 0$.

Proof. We split $\mathcal{T}-\widetilde{\mathcal{T}}_{h}$ into three terms

$$
\mathcal{T}-\widetilde{\mathcal{T}}_{h}=\left(\mathcal{S}-\widetilde{\mathcal{S}}_{h}\right) \gamma_{T} \mathcal{L} \mathcal{S}+\widetilde{\mathcal{S}}_{h} \gamma_{T} \mathcal{L}\left(\mathcal{S}-\widetilde{\mathcal{S}}_{h}\right)+\widetilde{\mathcal{S}}_{h} \gamma_{T}\left(\mathcal{L}-\mathcal{L}_{h}\right) \widetilde{\mathcal{S}}_{h}
$$

Given $\boldsymbol{f} \in \boldsymbol{L}_{t}^{2}(\Gamma)$, by definition, $\left(\mathcal{S}-\widetilde{\mathcal{S}}_{h}\right) \gamma_{T} \mathcal{L S} \boldsymbol{f}=\operatorname{curl}_{\Gamma} q-\operatorname{curl}_{\Gamma} q_{h}$ with $q$ and $q_{h}$ being the solution of $(2.2)$ and (2.5), respectively, with $\boldsymbol{\mu}=\gamma_{T} \mathcal{L} \mathcal{S} \boldsymbol{f}$. As shown in Lemma 2.11, $q \in H^{1+t}(\Gamma) / \mathbb{R}$ for $t<\min \left\{s_{\Gamma}, 1\right\}$. Hence we have that

$$
\left\|\operatorname{curl}_{\Gamma} q-\operatorname{curl}_{\Gamma} q_{h}\right\|_{0, \Gamma} \leqslant C h^{t}\|q\|_{H^{1+t}(\Gamma) / \mathbb{R}}
$$

Meanwhile, by the inequality (2.2) of [18] and (2.13), we obtain that

$$
\|p\|_{H^{1+t}(\Gamma) / \mathbb{R}} \leqslant C\left\|\operatorname{curl}_{\Gamma} \gamma_{T} \mathcal{L} \mathcal{S} \boldsymbol{f}\right\|_{0, \Gamma} \leqslant C\|\mathcal{S} \boldsymbol{f}\|_{0, \Gamma} \leqslant C\|\boldsymbol{f}\|_{0, \Gamma}
$$

Therefore,

$$
\left\|\left(\mathcal{S}-\widetilde{\mathcal{S}}_{h}\right) \gamma_{T} \mathcal{L}\right\| \leqslant C h^{t} \quad \text { and } \quad\left\|\left(\mathcal{S}-\widetilde{\mathcal{S}}_{h}\right) \gamma_{T} \mathcal{L} \mathcal{S}\right\| \leqslant C h^{t}
$$

For the second term of $(3.2)$, we consider its adjoint $\left(\widetilde{\mathcal{S}}_{h} \gamma_{T} \mathcal{L}\left(\mathcal{S}-\widetilde{\mathcal{S}}_{h}\right)\right)^{*}=\left(\mathcal{S}-\widetilde{\mathcal{S}}_{h}\right) \gamma_{T} \mathcal{L} \widetilde{\mathcal{S}}_{h}$. In fact, given $\boldsymbol{f}, \boldsymbol{g} \in \boldsymbol{L}_{t}^{2}(\Gamma)$,

$$
\begin{aligned}
& \left\langle\boldsymbol{f}, \widetilde{\mathcal{S}}_{h} \gamma_{T} \mathcal{L}\left(\mathcal{S}-\widetilde{\mathcal{S}}_{h}\right) \boldsymbol{g}\right\rangle=\left\langle\widetilde{\mathcal{S}}_{h} \boldsymbol{f}, \gamma_{T} \mathcal{L}\left(\mathcal{S}-\widetilde{\mathcal{S}}_{h}\right) \boldsymbol{g}\right\rangle=a\left(\mathcal{L} \widetilde{\mathcal{S}}_{h} \boldsymbol{f}, \mathcal{L}\left(\mathcal{S}-\widetilde{\mathcal{S}}_{h}\right) \boldsymbol{g}\right) \\
& \quad=\left\langle\gamma_{T} \mathcal{L} \widetilde{\mathcal{S}}_{h} \boldsymbol{f},\left(\mathcal{S}-\widetilde{\mathcal{S}}_{h}\right) \boldsymbol{g}\right\rangle=\left\langle\mathcal{S} \gamma_{T} \mathcal{L} \widetilde{\mathcal{S}}_{h} \boldsymbol{f}, \boldsymbol{g}\right\rangle-\left\langle\widetilde{\mathcal{S}}_{h} \gamma_{T} \mathcal{L} \widetilde{\mathcal{S}}_{h} \boldsymbol{f}, \boldsymbol{g}\right\rangle=\left\langle\left(\mathcal{S}-\widetilde{\mathcal{S}}_{h}\right) \gamma_{T} \mathcal{L} \widetilde{\mathcal{S}}_{h} \boldsymbol{f}, \boldsymbol{g}\right\rangle .
\end{aligned}
$$

Using the previous results, it holds that

$$
\left\|\widetilde{\mathcal{S}}_{h} \gamma_{T} \mathcal{L}\left(\mathcal{S}-\widetilde{\mathcal{S}}_{h}\right)\right\|=\left\|\left(\mathcal{S}-\widetilde{\mathcal{S}}_{h}\right) \gamma_{T} \mathcal{L} \widetilde{\mathcal{S}}_{h}\right\| \leqslant\left\|\left(\mathcal{S}-\widetilde{\mathcal{S}}_{h}\right) \gamma_{T} \mathcal{L}\right\|\left\|\widetilde{\mathcal{S}}_{h}\right\| \leqslant C h^{t} .
$$

To treat the third term of (3.2), we use the decomposition in Lemma 2.1, along with Lemmas 2.3, 2.8 and 2.4 to obtain

$$
\begin{aligned}
\left\|\widetilde{\mathcal{S}}_{h} \gamma_{T}\left(\mathcal{L}-\mathcal{L}_{h}\right) \widetilde{\mathcal{S}}_{h}\right\| & =\left\|\widetilde{\mathcal{S}}_{h} \gamma_{T}\left(\mathcal{L}-\mathcal{P} \mathcal{L}_{h}\right) \widetilde{\mathcal{S}}_{h}\right\| \leqslant C\left\|\left(\mathcal{L}-\mathcal{P} \mathcal{L}_{h}\right) \widetilde{\mathcal{S}}_{h}\right\| \\
& \leqslant C\left\|\left(\mathcal{L}-\mathcal{L}_{h}\right) \widetilde{\mathcal{S}}_{h}\right\|+C\left\|(I-\mathcal{P}) \mathcal{L}_{h} \widetilde{\mathcal{S}}_{h}\right\| \leqslant C h^{1 / 2}\left\|\widetilde{\mathcal{S}}_{h}\right\|+C h^{1 / 2+s}\left\|\mathcal{L}_{h} \widetilde{\mathcal{S}}_{h}\right\| \leqslant C h^{1 / 2}
\end{aligned}
$$

Putting all the terms together, we obtain that $\left\|\mathcal{T}-\mathcal{T}_{h}\right\| \leqslant C h^{\min \{1 / 2, t\}} \rightarrow 0$. 
Consequently, the eigenvalues of $\widetilde{\mathcal{T}}_{h}$ approximate that of $\mathcal{T}$ with correct multiplicities and there are no spurious modes. Next we apply the Babuška-Osborn theory [3] to show the convergence order of $\widetilde{\lambda}_{h}$. Let $\mu$ be a non-zero eigenvalue of $\mathcal{T}$ with multiplicity $m$ and $\widetilde{\mu}_{h,(j)}, j=1, \ldots, m$, be the eigenvalues of $\widetilde{\mathcal{T}}_{h}$ that approximate $\mu$. Due to Lemmas 2.11 and 3.1, let the eigenvalues $\lambda$ and $\widetilde{\lambda}_{h,(j)}$ of (2.1) and (3.1), respectively, are such that $\lambda=-\mu^{-1}$ and $\widetilde{\lambda}_{h,(j)}=-\widetilde{\mu}_{h,(j)}^{-1}$.

Let $\rho(\mathcal{T}) \subset \mathbb{C}$ be the resolvent set of $\mathcal{T}$. For a simple closed curve $\mathcal{C} \subset \rho(\mathcal{T})$ which encloses only one eigenvalue $\mu$ (with multiplicity $m$ ) of $\mathcal{T}$, we define a projection operator $E(\mu)$ by

$$
E(\mu)=\frac{1}{2 \pi i} \int_{\mathcal{C}}(z-\mathcal{T})^{-1} \mathrm{~d} z
$$

Let $\boldsymbol{f}_{1}, \ldots, \boldsymbol{f}_{m} \in E:=E(\mu) \boldsymbol{L}_{t}^{2}(\Gamma)$ be independent eigenvectors of $\mu$ with $\left\|\boldsymbol{f}_{i}\right\|_{0, \Gamma}=1$ for $i=1, \ldots, m$. Since $\mathcal{T}$ is self-adjoint, $E=\operatorname{span}\left\{\boldsymbol{f}_{1}, \ldots, \boldsymbol{f}_{m}\right\}$, i.e., the eigenspace associated to $\mu$. Define

$$
\widetilde{\lambda}_{h}^{\text {mean }}=\frac{1}{m} \sum_{j=1}^{m} \widetilde{\lambda}_{h,(j)}=-\frac{1}{m} \sum_{j=1}^{m} \widetilde{\mu}_{h,(j)}^{-1} .
$$

Theorem 3.3. For a fixed $\lambda \neq 0,\left|\lambda-\widetilde{\lambda}_{h}^{\text {mean }}\right| \leqslant C h^{2 \widetilde{r}}$, for any $\widetilde{r}<\min \left\{s_{\Gamma}, 1 / 2\right\}$.

Proof. The Babuška-Osborn theory ([3], Sect. 7) implies that

$$
\left|\lambda-\widetilde{\lambda}_{h}^{\text {mean }}\right| \leqslant C \sum_{i, j=1}^{m}\left|\left\langle\left(\mathcal{T}-\widetilde{\mathcal{T}}_{h}\right) \boldsymbol{f}_{i}, \boldsymbol{f}_{j}\right\rangle\right|+C\left\|\left.\left(\mathcal{T}-\widetilde{\mathcal{T}}_{h}\right)\right|_{E}\right\|^{2} .
$$

The bound of the second term is given by Theorem 3.2: $\left\|\left.\left(\mathcal{T}-\widetilde{\mathcal{T}}_{h}\right)\right|_{E}\right\|^{2} \leqslant\left\|\mathcal{T}-\widetilde{\mathcal{T}}_{h}\right\|^{2} \leqslant C h^{\min \{1,2 t\}}$, for $t<\min \left\{s_{\Gamma}, 1\right\}$. For the first term, we rewrite it as

$$
\begin{aligned}
\left\langle\left(\mathcal{T}-\widetilde{\mathcal{T}}_{h}\right) \boldsymbol{f}_{i}, \boldsymbol{f}_{j}\right\rangle= & \left\langle\left(\mathcal{T}-\widetilde{\mathcal{T}}_{h}\right)\left(I-\widetilde{\mathcal{S}}_{h}\right) \boldsymbol{f}_{i}, \boldsymbol{f}_{j}\right\rangle+\left\langle\left(\mathcal{T}-\widetilde{\mathcal{T}}_{h}\right) \widetilde{\mathcal{S}}_{h} \boldsymbol{f}_{i},\left(I-\widetilde{\mathcal{S}}_{h}\right) \boldsymbol{f}_{j}\right\rangle \\
& +\left\langle\left(\mathcal{T}-\widetilde{\mathcal{T}}_{h}\right) \widetilde{\mathcal{S}}_{h} \boldsymbol{f}_{i}, \widetilde{\mathcal{S}}_{h} \boldsymbol{f}_{j}\right\rangle .
\end{aligned}
$$

Noting that $\widetilde{\mathcal{S}}_{h} \mathcal{S}=\widetilde{\mathcal{S}}_{h}$ by Lemma 2.10 and $\boldsymbol{f}_{i}=-\lambda \mathcal{T} \boldsymbol{f}_{i}$, we follow the same argument as in Theorem 3.2 to see that $\left\|\left(I-\widetilde{\mathcal{S}}_{h}\right) \boldsymbol{f}_{i}\right\|_{0, \Gamma}=\left\|\lambda\left(\mathcal{S}-\widetilde{\mathcal{S}}_{h}\right) \gamma_{T} \mathcal{L} \mathcal{S} \boldsymbol{f}_{i}\right\| \leqslant C h^{t}\left\|\boldsymbol{f}_{i}\right\|_{0, \Gamma} \leqslant C h^{t}$. Here $C$ is dependent on $\lambda$. Consequently, by Theorem 3.2, we have that

$$
\left|\left\langle\left(\mathcal{T}-\widetilde{\mathcal{T}}_{h}\right)\left(I-\widetilde{\mathcal{S}}_{h}\right) \boldsymbol{f}_{i}, \boldsymbol{f}_{j}\right\rangle\right|+\left|\left\langle\left(\mathcal{T}-\widetilde{\mathcal{T}}_{h}\right) \widetilde{\mathcal{S}}_{h} \boldsymbol{f}_{i},\left(I-\widetilde{\mathcal{S}}_{h}\right) \boldsymbol{f}_{j}\right\rangle\right| \leqslant C h^{\min \{1 / 2+t, 2 t\}} .
$$

For the remaining term, we apply Lemma 2.10, the Galerkin orthogonality $a\left(\mathcal{L} \boldsymbol{f}-\mathcal{L}_{h} \boldsymbol{f}, \boldsymbol{v}_{h}\right)=0$ for all $\boldsymbol{v}_{h} \in V_{h}$ and Lemma 2.8 to deduce that

$$
\begin{aligned}
\left|\left\langle\left(\mathcal{T}-\widetilde{\mathcal{T}}_{h}\right) \widetilde{\mathcal{S}}_{h} \boldsymbol{f}_{i}, \widetilde{\mathcal{S}}_{h} \boldsymbol{f}_{j}\right\rangle\right| & =\left|\left\langle\mathcal{S} \gamma_{T} \mathcal{L} \widetilde{\mathcal{S}}_{h} \boldsymbol{f}_{i}-\widetilde{\mathcal{S}}_{h} \gamma_{T} \mathcal{L}_{h} \widetilde{\mathcal{S}}_{h} \boldsymbol{f}_{i}, \widetilde{\mathcal{S}}_{h} \boldsymbol{f}_{j}\right\rangle\right|=\left|\left\langle\gamma_{T} \mathcal{L} \widetilde{\mathcal{S}}_{h} \boldsymbol{f}_{i}-\gamma_{T} \mathcal{L}_{h} \widetilde{\mathcal{S}}_{h} \boldsymbol{f}_{i}, \widetilde{\mathcal{S}}_{h} \boldsymbol{f}_{j}\right\rangle\right| \\
& =\left|a\left(\mathcal{L} \widetilde{\mathcal{S}}_{h} \boldsymbol{f}_{i}-\mathcal{L}_{h} \widetilde{\mathcal{S}}_{h} \boldsymbol{f}_{i}, \mathcal{L} \widetilde{\mathcal{S}}_{h} \boldsymbol{f}_{j}\right)\right|=\left|a\left(\mathcal{L} \widetilde{\mathcal{S}}_{h} \boldsymbol{f}_{i}-\mathcal{L}_{h} \widetilde{\mathcal{S}}_{h} \boldsymbol{f}_{i}, \mathcal{L} \widetilde{\mathcal{S}}_{h} \boldsymbol{f}_{j}-\mathcal{L}_{h} \widetilde{\mathcal{S}}_{h} \boldsymbol{f}_{j}\right)\right| \\
& \leqslant C\left\|\mathcal{L}-\mathcal{L}_{h}\right\|^{2}\left\|\widetilde{\mathcal{S}}_{h}\right\|^{2} \leqslant C h .
\end{aligned}
$$

Altogether we obtain $\left|\lambda-\widetilde{\lambda}_{h}^{\text {mean }}\right| \leqslant C h^{\min \{1,2 t\}} . A s \min \left\{\min \left\{s_{\Gamma}, 1\right\}, 1 / 2\right\}=\min \left\{s_{\Gamma}, 1 / 2\right\}$, the desired estimate holds for $\widetilde{r}=\min \{t, 1 / 2\}$. 


\section{Finite element method USing $\mathcal{S}_{h}$}

Now consider the finite element eigenvalue problem of finding $\left(\lambda_{h}, \boldsymbol{u}_{h}\right) \in \mathbb{R} \times V_{h}$ such that

$$
a\left(\boldsymbol{u}_{h}, \boldsymbol{v}_{h}\right)=-\lambda_{h}\left\langle\mathcal{S}_{h} \boldsymbol{u}_{h, T}, \boldsymbol{v}_{h, T}\right\rangle, \quad \forall \boldsymbol{v}_{h} \in V_{h},
$$

where $\mathcal{S}_{h}$ is defined in (2.6). We start with the well-posedness of the corresponding source problem: for $\boldsymbol{f} \in \boldsymbol{L}_{t}^{2}(\Gamma)$, find $\boldsymbol{u}_{h} \in V_{h}$ such that

$$
a\left(\boldsymbol{u}_{h}, \boldsymbol{v}_{h}\right)=\left\langle\mathcal{S}_{h} \boldsymbol{f}, \boldsymbol{v}_{h, T}\right\rangle, \quad \forall \boldsymbol{v}_{h} \in V_{h} .
$$

In other words, to find $\boldsymbol{u}_{h}=\mathcal{L}_{h} \mathcal{S}_{h} \boldsymbol{f}$. Formally, we characterize $\boldsymbol{u}_{h}$ as the solution of the operator equation

$$
\left(I+\mathcal{K}_{h}\right) \boldsymbol{u}_{h}=\mathcal{L}_{h}^{+} \mathcal{S}_{h} \boldsymbol{f} .
$$

Using the discrete decomposition $V_{h}=Z_{h} \oplus \nabla\left(U_{h} / \mathbb{R}\right)$, we can show the equivalence between (4.2) and (4.3) (see e.g., [15], Lem. 3.1).

Lemma 4.1. $\boldsymbol{u}_{h} \in V_{h}$ is a solution of (4.2) if and only if $\boldsymbol{u}_{h} \in \boldsymbol{L}^{2}(\Omega)$ is a solution of (4.3).

In what follows, we deal with the existence and (uniform) continuous dependence on $\boldsymbol{f} \in \boldsymbol{L}_{t}^{2}(\Gamma)$ of $\boldsymbol{u}_{h}$. In (4.3), the uniform boundedness of $\left(I+\mathcal{K}_{h}\right)^{-1}$ from $\boldsymbol{L}^{2}(\Omega)$ to $\boldsymbol{L}^{2}(\Omega)$ is guaranteed by the collective compactness of $\left\{\mathcal{K}_{h}\right\}_{h}$. Consequently, the operator $\left(I+\mathcal{K}_{h}\right)^{-1}=I-\mathcal{K}_{h}\left(I+\mathcal{K}_{h}\right)^{-1}$ confined on $V_{h}$ is also uniformly bounded from $V_{h}$ to $V_{h}$. Hence it requires the uniform boundedness of $\mathcal{L}_{h}^{+} \mathcal{S}_{h}$ on $\boldsymbol{L}_{t}^{2}(\Gamma)$ to show the well-posedness of $\boldsymbol{u}_{h}$. However, what's in hand now is only the well-posedness of $\mathcal{L}_{h}^{+}$on $\boldsymbol{H}\left(\operatorname{div}_{\Gamma}^{0} ; \Gamma\right)$, while the range of $\mathcal{S}_{h}$ is not contained in $\boldsymbol{H}\left(\operatorname{div}_{\Gamma}^{0} ; \Gamma\right)$. Noticing in the equation of $\mathcal{L}_{h}^{+} \mathcal{S}_{h}$,

$$
a_{+}\left(\mathcal{L}_{h}^{+} \mathcal{S}_{h} \boldsymbol{f}, \boldsymbol{v}_{h}\right)=\left\langle\mathcal{S}_{h} \boldsymbol{f}, \boldsymbol{v}_{h, T}\right\rangle, \quad \forall \boldsymbol{v}_{h} \in V_{h},
$$

the sesquilinear $a_{+}(\cdot, \cdot)$ is coercive, hence what remains is to show the uniform boundedness of the right-handside $\left\langle\mathcal{S}_{h} \boldsymbol{f}, \boldsymbol{v}_{h, T}\right\rangle$ for $\boldsymbol{v}_{h} \in V_{h}$.

Lemma 4.2. $\mathcal{L}_{h}^{+} \mathcal{S}_{h}$ and $\mathcal{L}_{h} \mathcal{S}_{h}$ are well-defined and uniformly bounded on $\boldsymbol{L}_{t}^{2}(\Gamma)$.

Proof. Note that

$$
\left|\left\langle\mathcal{S}_{h} \boldsymbol{f}, \boldsymbol{v}_{h, T}\right\rangle\right|=\left|\left\langle\boldsymbol{f}, \mathcal{S}_{h} \boldsymbol{v}_{h, T}\right\rangle\right| \leqslant\|\boldsymbol{f}\|_{0, \Gamma}\left\|\mathcal{S}_{h} \boldsymbol{v}_{h, T}\right\|_{0, \Gamma}
$$

Decompose $\boldsymbol{v}_{h}$ into $\boldsymbol{v}_{h}=\boldsymbol{z}_{h}+\nabla p_{h}$ according to $V_{h}=Z_{h} \oplus \nabla\left(U_{h} / \mathbb{R}\right)$ and $\boldsymbol{z}_{h}$ into $\boldsymbol{z}_{h}=\boldsymbol{z}_{h, 0}+\nabla p^{h}$ according to $\boldsymbol{H}(\mathbf{c u r l} ; \Omega)=Z \oplus \nabla\left(H^{1}(\Omega) / \mathbb{R}\right)$. It is easily seen that $\gamma_{T} \nabla p^{h}=\boldsymbol{z}_{h, T}-\boldsymbol{z}_{h, 0, T} \in \boldsymbol{L}_{t}^{2}(\Gamma)$. We have

$$
\left\|\mathcal{S}_{h} \boldsymbol{v}_{h, T}\right\|_{0, \Gamma}=\left\|\mathcal{S}_{h} \boldsymbol{z}_{h, T}\right\|_{0, \Gamma} \leqslant\left\|\mathcal{S}_{h} \boldsymbol{z}_{h, 0, T}\right\|_{0, \Gamma}+\left\|\mathcal{S}_{h} \gamma_{T} \nabla p^{h}\right\|_{0, \Gamma} .
$$

On one hand, by Lemma 2.3, we have that

$$
\left\|\mathcal{S}_{h} \boldsymbol{z}_{h, 0, T}\right\|_{0, \Gamma} \leqslant\left\|\boldsymbol{z}_{h, 0, T}\right\|_{0, \Gamma} \leqslant C\left\|\boldsymbol{z}_{h, 0}\right\|_{\operatorname{curl}, \Omega} \leqslant C\left\|\boldsymbol{z}_{h}\right\|_{\operatorname{curl}, \Omega} \leqslant C\left\|\boldsymbol{v}_{h}\right\|_{\operatorname{curl}, \Omega} .
$$

On the other hand, using the identity $\left(I-\pi_{h}^{1}\right) \nabla p^{h}+\left(I-\pi_{h}^{1}\right) z_{h, 0}=\left(I-\pi_{h}^{1}\right) z_{h}=0, \pi_{h}^{1} \nabla p^{h}$ is well-defined, and it holds

$$
\left\|\mathcal{S}_{h} \gamma_{T} \nabla p^{h}\right\|_{0, \Gamma} \leqslant\left\|\mathcal{S}_{h} \gamma_{T} \pi_{h}^{1} \nabla p^{h}\right\|_{0, \Gamma}+\left\|\mathcal{S}_{h} \gamma_{T}\left(I-\pi_{h}^{1}\right) \boldsymbol{z}_{h, 0}\right\|_{0, \Gamma} .
$$

Since $\operatorname{curl} \pi_{h}^{1} \nabla p^{h}=\pi_{h}^{2} \operatorname{curl} \nabla p^{h}=0$ and $\pi_{h}^{1} \nabla p^{h} \in V_{h}$, there exists $\phi_{h} \in U_{h}$ such that $\pi_{h}^{1} \nabla p^{h}=\nabla q_{h}$, which implies there is $q_{h}=\left.\phi_{h}\right|_{\Gamma} \in U_{h}^{\Gamma}$ and $\mathcal{S}_{h} \gamma_{T} \pi_{h}^{1} \nabla p^{h}=\mathcal{S}_{h} \nabla_{\Gamma} q_{h}=0$. In addition, we have that

$$
\left\|\mathcal{S}_{h} \gamma_{T}\left(I-\pi_{h}^{1}\right) \boldsymbol{z}_{h, 0}\right\|_{0, \Gamma}^{2} \leqslant\left\|\gamma_{T}\left(I-\pi_{h}^{1}\right) \boldsymbol{z}_{h, 0}\right\|_{0, \Gamma}^{2} \leqslant\left\|\left(I-\pi_{h}^{1}\right) \boldsymbol{z}_{h, 0}\right\|_{0, \Gamma}^{2}=\sum_{F \in \tau_{h}(\Gamma)}\left\|\left(I-\pi_{h}^{1}\right) \boldsymbol{z}_{h, 0}\right\|_{0, F}^{2},
$$


B. GONG

where $F$ is a face of an element on the boundary. Let $K$ be the element containing $F$. Noting that $\boldsymbol{z}_{h, 0} \in$ $\boldsymbol{H}^{1 / 2+s}(\Omega)$ and $\operatorname{curl} \boldsymbol{z}_{h, 0} \in W_{h}$, we map $\left(I-\pi_{h}^{1}\right) \boldsymbol{z}_{h, 0}$ from the element $K$ to the reference element $\hat{K}$ then map back to see that

$$
\left\|\left(I-\pi_{h}^{1}\right) \boldsymbol{z}_{h, 0}\right\|_{0, F}^{2} \leqslant C h^{s}\left(\left\|\boldsymbol{z}_{h, 0}\right\|_{1 / 2+s, K}+\left\|\operatorname{curl} \boldsymbol{z}_{h, 0}\right\|_{0, K}\right) \leqslant C h^{s}\left\|\boldsymbol{z}_{h, 0}\right\|_{\mathbf{c u r l}, K} \leqslant C h^{s}\left\|\boldsymbol{v}_{h}\right\|_{\mathbf{c u r l}, K} .
$$

Altogether we obtain that

$$
\begin{aligned}
\left\|\mathcal{S}_{h} \gamma_{T} \nabla p^{h}\right\|_{0, \Gamma} & \leqslant C h^{s}\left\|\boldsymbol{v}_{h}\right\|_{\mathbf{c u r l}, \Omega}, \\
\left\|\mathcal{S}_{h} \boldsymbol{v}_{h, T}\right\|_{0, \Gamma} & \leqslant C\left\|\boldsymbol{v}_{h}\right\|_{\mathbf{c u r l}, \Omega}, \quad \text { and } \quad\left|\left\langle\mathcal{S}_{h} \boldsymbol{f}, \boldsymbol{v}_{h, T}\right\rangle\right| \leqslant C\|\boldsymbol{f}\|_{0, \Gamma}\left\|\boldsymbol{v}_{h}\right\|_{\mathbf{c u r l}, \Omega} .
\end{aligned}
$$

Using the coercivity and boundedness of $a_{+}(\cdot, \cdot)$, we obtain the well-posedness of $\mathcal{L}_{h}^{+} \mathcal{S}_{h}$ on $\boldsymbol{L}_{t}^{2}(\Gamma)$ with $\left\|\mathcal{L}_{h}^{+} \mathcal{S}_{h}\right\| \leqslant$ C. Due to Lemmas 4.1 and 2.7, (4.3) and (4.2) are well-posed. Moreover,

$$
\mathcal{L}_{h} \mathcal{S}_{h}=\left(I+\mathcal{K}_{h}\right)^{-1} \mathcal{L}_{h}^{+} \mathcal{S}_{h}
$$

and $\left\|\mathcal{L}_{h} \mathcal{S}_{h}\right\| \leqslant C$. The proof is complete.

We now can define the solution operator $\mathcal{T}_{h}: \boldsymbol{L}_{t}^{2}(\Gamma) \rightarrow \boldsymbol{L}_{t}^{2}(\Gamma)$ for (4.1) by

$$
\mathcal{T}_{h}=\mathcal{S}_{h} \gamma_{T} \mathcal{L}_{h} \mathcal{S}_{h}
$$

Lemma 4.3. $\mathcal{T}_{h}$ are uniformly bounded and self-adjoint. There is a bijection between the eigenpairs of (4.1) and those of $\mathcal{T}_{h}$ for nonzero eigenvalues.

Proof. Due to Lemma 4.2, $\mathcal{T}_{h}$ are uniformly bounded with

$$
\left\|\mathcal{T}_{h}\right\| \leqslant\left\|\mathcal{S}_{h} \gamma_{T} \mathcal{P} \mathcal{L}_{h} \mathcal{S}_{h}\right\|+\left\|\mathcal{S}_{h} \gamma_{T}(I-\mathcal{P}) \mathcal{L}_{h} \mathcal{S}_{h}\right\| \leqslant C+C h^{s} \leqslant C
$$

The self-adjoint property and the correspondence between the eigenpairs of $\mathcal{T}_{h}$ and (4.1) can be shown the same way as in Lemma 2.11.

The proof of $\mathcal{T}_{h}$ converging to $\mathcal{T}$ and $\lambda_{h}$ converging to $\lambda$ follows similar steps in that of Theorems 3.2 and 3.3, respectively. However, there are several technical differences.

Theorem 4.4. $\left\|\mathcal{T}-\mathcal{T}_{h}\right\| \rightarrow 0$.

Proof. Note that

$$
\mathcal{T}-\mathcal{T}_{h}=\left(\mathcal{S}-\mathcal{S}_{h}\right) \gamma_{T} \mathcal{L} \mathcal{S}+\mathcal{S}_{h} \gamma_{T}\left(\mathcal{L}-\mathcal{L}_{h}\right) \mathcal{S}+\mathcal{S}_{h} \gamma_{T} \mathcal{L}_{h}\left(\mathcal{S}-\mathcal{S}_{h}\right)
$$

Given $\boldsymbol{f} \in \boldsymbol{L}_{t}^{2}(\Gamma)$, by definition, $\left(\mathcal{S}-\mathcal{S}_{h}\right) \gamma_{T} \mathcal{L S} \boldsymbol{f}=\nabla_{\Gamma} p-\nabla_{\Gamma} p_{h}$ with $p$ and $p_{h}$ being, respectively, the solution of (2.3) and (2.6) with $\boldsymbol{\mu}=\gamma_{T} \mathcal{L} \mathcal{S} \boldsymbol{f}$. By Lemma 2.3 and the proof of Lemma 2.11, we have that $\nabla_{\Gamma} p=$ $(\mathcal{S}-I) \gamma_{T} \mathcal{L} \mathcal{S} \boldsymbol{f} \in \boldsymbol{H}_{-}^{r}(\Gamma)$ for $r<\min \left\{\min \left\{s_{\Omega}, 1 / 2\right\}, \min \left\{s_{\Gamma}, 1\right\}\right\}=\min \left\{s_{\Omega}, s_{\Gamma}\right\}$ with

$$
\begin{aligned}
\left\|\nabla_{\Gamma} p\right\|_{r,-, \Gamma} & \leqslant\left\|\gamma_{T} \mathcal{L} \mathcal{S} \boldsymbol{f}\right\|_{r,-, \Gamma}+\left\|\mathcal{S} \gamma_{T} \mathcal{L} \mathcal{S} \boldsymbol{f}\right\|_{r,-, \Gamma} \\
& \leqslant C\|\mathcal{L} \mathcal{S} \boldsymbol{f}\|_{1 / 2+r, \Omega}+C\left\|\operatorname{curl}_{\Gamma} \gamma_{T} \mathcal{L} \mathcal{S} \boldsymbol{f}\right\|_{0, \Gamma} \leqslant C\|\boldsymbol{f}\|_{0, \Gamma} .
\end{aligned}
$$

Due to (17) of [8], it holds that

$$
\left\|\nabla_{\Gamma} p-\nabla_{\Gamma} p_{h}\right\|_{0, \Gamma} \leqslant C h^{r}\|p\|_{H^{1+r}(\Gamma) / \mathbb{R}} \leqslant C h^{r}\left\|\nabla_{\Gamma} p\right\|_{r,-, \Gamma} \leqslant C h^{r}\|\boldsymbol{f}\|_{0, \Gamma},
$$


which implies that $\left\|\left(\mathcal{S}-\mathcal{S}_{h}\right) \gamma_{T} \mathcal{L S}\right\| \leqslant C h^{r}$.

For the third term of $(4.5)$, we consider its adjoint $\left(\mathcal{S}-\mathcal{S}_{h}\right) \gamma_{T} \mathcal{L}_{h} \mathcal{S}_{h}=\left(\mathcal{S}_{h} \gamma_{T} \mathcal{L}_{h}\left(\mathcal{S}-\mathcal{S}_{h}\right)\right)^{*}$. Given $\boldsymbol{f} \in \boldsymbol{L}_{t}^{2}(\Gamma)$, we write

$$
\left(\mathcal{S}-\mathcal{S}_{h}\right) \gamma_{T} \mathcal{L}_{h} \mathcal{S}_{h} \boldsymbol{f}=\left(\mathcal{S}-\mathcal{S}_{h}\right) \gamma_{T} \mathcal{P} \mathcal{L}_{h} \mathcal{S}_{h} \boldsymbol{f}+\left(\mathcal{S}-\mathcal{S}_{h}\right) \gamma_{T}(I-\mathcal{P}) \mathcal{L}_{h} \mathcal{S}_{h} \boldsymbol{f}
$$

By definition, $\left(\mathcal{S}-\mathcal{S}_{h}\right) \gamma_{T} \mathcal{P} \mathcal{L}_{h} \mathcal{S}_{h} \boldsymbol{f}=\nabla_{\Gamma} \phi-\nabla_{\Gamma} \phi_{h}$, where $\phi$ and $\phi_{h}$ are the solutions of (2.3) and (2.6) with $\boldsymbol{\mu}=\gamma_{T} \mathcal{P} \mathcal{L}_{h} \mathcal{S}_{h} \boldsymbol{f}$, respectively. Similarly, we have $\nabla_{\Gamma} \phi=(\mathcal{S}-I) \gamma_{T} \mathcal{P} \mathcal{L}_{h} \mathcal{S}_{h} \boldsymbol{f} \in \boldsymbol{H}_{-}^{r}(\Gamma)$ for $r<$ $\min \left\{\min \left\{s_{\Omega}, 1 / 2\right\}, \min \left\{s_{\Gamma}, 1 / 2\right\}\right\}=\min \left\{s_{\Omega}, s_{\Gamma}\right\}$ (here $r$ is assigned with the same value as in the above paragraph) with

$$
\begin{aligned}
\left\|\nabla_{\Gamma} \phi\right\|_{r,-, \Gamma} & \leqslant\left\|\gamma_{T} \mathcal{P} \mathcal{L}_{h} \mathcal{S} \boldsymbol{f}\right\|_{r,-, \Gamma}+\left\|\mathcal{S} \gamma_{T} \mathcal{P} \mathcal{L}_{h} \mathcal{S} \boldsymbol{f}\right\|_{r,-, \Gamma} \\
& \leqslant C\left\|\mathcal{P} \mathcal{L}_{h} \mathcal{S}_{h} \boldsymbol{f}\right\|_{1 / 2+r, \Omega}+C\left\|\operatorname{curl}_{\Gamma} \gamma_{T} \mathcal{P} \mathcal{L}_{h} \mathcal{S}_{h} \boldsymbol{f}\right\|_{-1 / 2, \Gamma} \\
& \leqslant C\left\|\mathcal{P} \mathcal{L}_{h} \mathcal{S}_{h} \boldsymbol{f}\right\|_{\text {curl }, \Omega}+C\left\|\mathcal{P} \mathcal{L}_{h} \mathcal{S}_{h} \boldsymbol{f}\right\|_{\text {curl }, \Omega} \leqslant C\|\boldsymbol{f}\|_{0, \Gamma}
\end{aligned}
$$

Therefore,

$$
\left\|\nabla_{\Gamma} \phi-\nabla_{\Gamma} \phi_{h}\right\|_{0, \Gamma} \leqslant C h^{r}\|\phi\|_{H^{1+r}(\Gamma) / \mathbb{R}} \leqslant C h^{r}\left\|\nabla_{\Gamma} \phi\right\|_{r,-, \Gamma} \leqslant C h^{r}\|\boldsymbol{f}\|_{0, \Gamma}
$$

Meanwhile, using (4.4), we have for $s \in\left(r, s_{\Omega}\right)$ that

$$
\left\|\left(\mathcal{S}-\mathcal{S}_{h}\right) \gamma_{T}(I-\mathcal{P}) \mathcal{L}_{h} \mathcal{S}_{h} \boldsymbol{f}\right\|_{0, \Gamma}=\left\|\mathcal{S}_{h} \gamma_{T}(I-\mathcal{P}) \mathcal{L}_{h} \mathcal{S}_{h} \boldsymbol{f}\right\|_{0, \Gamma} \leqslant C h^{s}\left\|\mathcal{L}_{h} \mathcal{S}_{h} \boldsymbol{f}\right\|_{\text {curl }, \Omega} \leqslant C h^{s}\|\boldsymbol{f}\|_{0, \Gamma} .
$$

Therefore, $\left\|\mathcal{S}_{h} \gamma_{T} \mathcal{L}_{h}\left(\mathcal{S}-\mathcal{S}_{h}\right)\right\|=\left\|\left(\mathcal{S}-\mathcal{S}_{h}\right) \gamma_{T} \mathcal{L}_{h} \mathcal{S}_{h}\right\| \leqslant C h^{r}$.

Similar to the proof of Theorem 3.2, we insert a $\mathcal{P}$ into the second term of (4.5) to obtain that

$$
\left\|\mathcal{S}_{h} \gamma_{T}\left(\mathcal{L}-\mathcal{L}_{h}\right) \mathcal{S}\right\| \leqslant\left\|\mathcal{S}_{h} \gamma_{T}\left(\mathcal{L}-\mathcal{P} \mathcal{L}_{h}\right) \mathcal{S}\right\|+\left\|\mathcal{S}_{h} \gamma_{T}(\mathcal{P}-I) \mathcal{L}_{h} \mathcal{S}\right\| \leqslant C h^{1 / 2}+C h^{s} \leqslant C h^{s},
$$

where we have used again (4.4). Altogether, we obtain $\left\|\mathcal{T}-\mathcal{T}_{h}\right\| \leqslant C h^{r} \rightarrow 0$.

Now we show the convergence order of $\lambda_{h}$. Let $\mu$ be a nonzero eigenvalue of $\mathcal{T}$ with multiplicity $m$ and $\mu_{h,(j)}, j=1, \ldots, m$, be the eigenvalues of $\mathcal{T}_{h}$ that approximate $\mu$. Let $\lambda$ and $\lambda_{h,(j)}$ be, respectively, the related eigenvalues of (2.1) and (4.1) with $\lambda=-\mu^{-1}$ and $\lambda_{h,(j)}=-\mu_{h,(j)}^{-1}$. Define

$$
\lambda_{h}^{\text {mean }}=\frac{1}{m} \sum_{j=1}^{m} \lambda_{h,(j)}=-\frac{1}{m} \sum_{j=1}^{m} \mu_{h,(j)}^{-1} .
$$

Theorem 4.5. For a fixed $\lambda \neq 0,\left|\lambda-\lambda_{h}^{\text {mean }}\right| \leqslant C h^{2 r}$, for any $r<\min \left\{s_{\Omega}, s_{\Gamma}\right\}$.

Proof. By the Babuška-Osborn theory,

$$
\left|\lambda-\lambda_{h}^{\text {mean }}\right| \leqslant C \sum_{i, j=1}^{m}\left|\left\langle\left(\mathcal{T}-\mathcal{T}_{h}\right) \boldsymbol{f}_{i}, \boldsymbol{f}_{j}\right\rangle\right|+C\left\|\left.\left(\mathcal{T}-\mathcal{T}_{h}\right)\right|_{E}\right\|^{2} .
$$

It holds that

$$
\begin{aligned}
\left\langle\left(\mathcal{T}-\mathcal{T}_{h}\right) \boldsymbol{f}_{i}, \boldsymbol{f}_{j}\right\rangle & =\left\langle\mathcal{S} \gamma_{T} \mathcal{L} \mathcal{S} \boldsymbol{f}_{i}, \boldsymbol{f}_{j}\right\rangle-\left\langle\mathcal{S}_{h} \gamma_{T} \mathcal{L}_{h} \mathcal{S}_{h} \boldsymbol{f}_{i}, \boldsymbol{f}_{j}\right\rangle \\
& =\left\langle\gamma_{T} \mathcal{L} \mathcal{S} \boldsymbol{f}_{i}, \mathcal{S} \boldsymbol{f}_{j}\right\rangle-\left\langle\gamma_{T} \mathcal{L}_{h} \mathcal{S}_{h} \boldsymbol{f}_{i}, \mathcal{S}_{h} \boldsymbol{f}_{j}\right\rangle=\left\langle\gamma_{T}\left(\mathcal{L}-\mathcal{L}_{h}\right) \boldsymbol{f}_{i}, \boldsymbol{f}_{j}\right\rangle \\
& =a\left(\left(\mathcal{L}-\mathcal{L}_{h}\right) \boldsymbol{f}_{i}, \mathcal{L} \boldsymbol{f}_{j}\right)=a\left(\left(\mathcal{L}-\mathcal{L}_{h}\right) \boldsymbol{f}_{i},\left(\mathcal{L}-\mathcal{L}_{h}\right) \boldsymbol{f}_{j}\right)
\end{aligned}
$$

where we have used Lemma $2.10, \boldsymbol{f}_{i}=-\lambda \mathcal{T} \boldsymbol{f}_{i}$, and the Galerkin orthogonality. By Lemma 2.8, $\left|\left\langle\left(\mathcal{T}-\mathcal{T}_{h}\right) \boldsymbol{f}_{i}, \boldsymbol{f}_{j}\right\rangle\right| \leqslant C h$. Using Theorem 4.4, we have that $\left\|\left.\left(\mathcal{T}-\mathcal{T}_{h}\right)\right|_{E}\right\| \leqslant\left\|\mathcal{T}-\mathcal{T}_{h}\right\| \leqslant C h^{r}$. Therefore, we conclude from (4.6) that $\left|\lambda-\lambda_{h}^{\text {mean }}\right| \leqslant C h^{2 r}$.

Comparing the results of Theorems 3.3 and 4.5 we see that the convergence order we obtain for $\lambda_{h}$ is not greater than that of $\widetilde{\lambda}_{h}$. 
B. GONG

\section{Additional approximation properties}

In this section we assume the parameters $\kappa$ and $\epsilon_{r}$ are such that $a(\cdot, \cdot)$ is positive definite on $\boldsymbol{H}(\mathbf{c u r l} ; \Omega) \times$ $\boldsymbol{H}(\mathbf{c u r l} ; \Omega)$. Define an operator $\mathbb{T}: \boldsymbol{H}(\operatorname{curl} ; \Omega) \rightarrow \boldsymbol{H}(\operatorname{curl} ; \Omega)$ by

$$
\mathbb{T}=\mathcal{L} \mathcal{S} \gamma_{T}
$$

It can be easily shown that there's a bijection between the eigenpairs of $\mathbb{T}$ and those of (2.1) for nonzero eigenvalues. The operator $\mathbb{T}$ is also compact since the range of $\mathbb{T}$ is contained in $\boldsymbol{H}^{1 / 2}(\mathbf{c u r l} ; \Omega)=\left\{\boldsymbol{u} \in \boldsymbol{H}^{1 / 2}(\Omega) \mid \mathbf{c u r l} \boldsymbol{u} \in\right.$ $\left.\boldsymbol{H}^{1 / 2}(\Omega)\right\}$ (see e.g., [15], Lem. 2.7), which is compactly embedded in $\boldsymbol{H}(\mathbf{c u r l} ; \Omega)$. Moreover, $\mathbb{T}$ is self-adjoint with respect to the inner product induced by $a(\cdot, \cdot)$, as

$$
a(\mathbb{T} \boldsymbol{u}, \boldsymbol{v})=\left\langle\mathcal{S} \boldsymbol{u}_{T}, \boldsymbol{v}_{T}\right\rangle=\left\langle\boldsymbol{u}_{T}, \mathcal{S} \boldsymbol{v}_{T}\right\rangle=a(\boldsymbol{u}, \mathbb{T} \boldsymbol{v}),
$$

for $\boldsymbol{u}, \boldsymbol{v} \in \boldsymbol{H}(\mathbf{c u r l} ; \Omega)$. Consider an auxiliary eigenvalue problem of finding $\left(\lambda_{h}^{0}, \boldsymbol{u}_{h}\right) \in \mathbb{R} \times V_{h}$ such that

$$
a\left(\boldsymbol{u}_{h}, \boldsymbol{v}_{h}\right)=-\lambda_{h}^{0}\left\langle\mathcal{S} \boldsymbol{u}_{h, T}, \boldsymbol{v}_{h, T}\right\rangle, \quad \forall \boldsymbol{v}_{h} \in V_{h}
$$

Then for (3.1), (4.1) and (5.1) we define the corresponding solution operators as $\widetilde{\mathbb{T}}_{h}: V_{h} \rightarrow V_{h}, \mathbb{T}_{h}: V_{h} \rightarrow V_{h}$ and $\mathbb{T}_{h}^{0}: V_{h} \rightarrow V_{h}$ respectively, such that

$$
\widetilde{\mathbb{T}}_{h}=\mathcal{L}_{h} \widetilde{\mathcal{S}}_{h} \gamma_{T}, \quad \mathbb{T}_{h}=\mathcal{L}_{h} \mathcal{S}_{h} \gamma_{T}, \quad \mathbb{T}_{h}^{0}=\mathcal{L}_{h} \mathcal{S} \gamma_{T}
$$

Similarly, we can show that $\widetilde{\mathbb{T}}_{h}, \mathbb{T}_{h}$ and $\mathbb{T}_{h}^{0}$ are bounded, self-adjoint with respect to the inner product induced by $a(\cdot, \cdot)$, and there are respectively bijections between eigenpairs of these operators and those of (3.1), (4.1) and (5.1).

Let $\lambda_{j}, \lambda_{h, j}, \widetilde{\lambda}_{h, j}$ and $\lambda_{h, j}^{0}$ be the $j$-th eigenvalues in descending order of (2.1), (4.1), (3.1) and (5.1), respectively. Namely, $\lambda_{j} \geqslant \lambda_{j+1}, \lambda_{h, j} \geqslant \lambda_{h, j+1}, \widetilde{\lambda}_{h, j} \geqslant \widetilde{\lambda}_{h, j+1}$ and $\lambda_{h, j}^{0} \geqslant \lambda_{h, j+1}^{0}$ for $j=1,2, \ldots$ Under the setting of this section $\lambda_{j}, \lambda_{h, j}, \widetilde{\lambda}_{h, j}$ and $\lambda_{h, j}^{0}$ are negative. Let $\mu_{j}, \mu_{h, j}, \widetilde{\mu}_{h, j}$ and $\mu_{h, j}^{0}$ be the eigenvalues of $\mathbb{T}, \mathbb{T}_{h}, \widetilde{\mathbb{T}}_{h}$ and $\mathbb{T}_{h}^{0}$ corresponding to $\lambda_{j}, \lambda_{h, j}, \widetilde{\lambda}_{h, j}$ and $\lambda_{h, j}^{0}$, respectively, i.e., $\lambda_{j}=-\mu_{j}^{-1}, \lambda_{h, j}=-\mu_{h, j}^{-1}, \widetilde{\lambda}_{h, j}=-\widetilde{\mu}_{h, j}^{-1}$ and $\lambda_{h, j}^{0}=-\left(\mu_{h, j}^{0}\right)^{-1}$. Denote by $V^{(j)}$ the set of all subspaces of $\boldsymbol{H}(\mathbf{c u r l} ; \Omega)$ with $\operatorname{dimension} j$, and by $V_{h}^{(j)}$ the set of all subspaces of $V_{h}$ with dimension $j$. Then, for $\lambda_{j}$ we have the min-max property

$$
\lambda_{j}=-\mu_{j}^{-1}=-\left(\max _{E \in V^{(j)}} \min _{\boldsymbol{u} \in E} a(\mathbb{T} \boldsymbol{u}, \boldsymbol{u}) / a(\boldsymbol{u}, \boldsymbol{u})\right)^{-1}=-\min _{E \in V^{(j)}} \max _{\boldsymbol{u} \in E} a(\boldsymbol{u}, \boldsymbol{u}) /\left\langle\mathcal{S} \boldsymbol{u}_{T}, \boldsymbol{u}_{T}\right\rangle .
$$

Similarly, the min-max property holds for $\lambda_{h, j}, \widetilde{\lambda}_{h, j}$ and $\lambda_{h, j}^{0}$,

$$
\begin{aligned}
& \lambda_{h, j}=-\min _{E_{h} \in V_{h}^{(j)}} \max _{\boldsymbol{u}_{h} \in E_{h}} a\left(\boldsymbol{u}_{h}, \boldsymbol{u}_{h}\right) /\left\langle\mathcal{S}_{h} \boldsymbol{u}_{h, T}, \boldsymbol{u}_{h, T}\right\rangle, \\
& \widetilde{\lambda}_{h, j}=-\min _{E_{h} \in V_{h}^{(j)}} \max _{\boldsymbol{u}_{h} \in E_{h}} a\left(\boldsymbol{u}_{h}, \boldsymbol{u}_{h}\right) /\left\langle\widetilde{\mathcal{S}}_{h} \boldsymbol{u}_{h, T}, \boldsymbol{u}_{h, T}\right\rangle, \\
& \lambda_{h, j}^{0}=-\min _{E_{h} \in V_{h}^{(j)}} \max _{\boldsymbol{u}_{h} \in E_{h}} a\left(\boldsymbol{u}_{h}, \boldsymbol{u}_{h}\right) /\left\langle\mathcal{S} \boldsymbol{u}_{h, T}, \boldsymbol{u}_{h, T}\right\rangle .
\end{aligned}
$$

If there is no ambiguity we shall omit the subscript $\cdot_{j}$, and use $\lambda, \lambda_{h}, \widetilde{\lambda}_{h}, \lambda_{h}^{0}$ to represent $\lambda_{j}, \lambda_{h, j}, \widetilde{\lambda}_{h, j}, \lambda_{h, j}^{0}$ with the same fixed $j$. 
Lemma 5.1. $\lambda_{h} \geqslant \lambda_{h}^{0} \geqslant \widetilde{\lambda}_{h}$.

Proof. Given $\boldsymbol{f} \in \boldsymbol{L}_{t}^{2}(\Gamma)$, by Lemma 2.10, we have that

$$
\begin{aligned}
\left\langle\mathcal{S}_{h} \boldsymbol{f}, \boldsymbol{f}\right\rangle-\langle\mathcal{S} \boldsymbol{f}, \boldsymbol{f}\rangle & =\left\langle\mathcal{S}_{h} \boldsymbol{f}, \boldsymbol{f}\right\rangle-\langle\mathcal{S} \boldsymbol{f}, \boldsymbol{f}\rangle-\langle\boldsymbol{f}, \mathcal{S} \boldsymbol{f}\rangle+\langle\mathcal{S} \boldsymbol{f}, \mathcal{S} \boldsymbol{f}\rangle \\
& =\left\langle\mathcal{S}_{h} \boldsymbol{f}, \mathcal{S}_{h} \boldsymbol{f}\right\rangle-\left\langle\mathcal{S} \boldsymbol{f}, \mathcal{S}_{h} \boldsymbol{f}\right\rangle-\left\langle\mathcal{S}_{h} \boldsymbol{f}, \mathcal{S} \boldsymbol{f}\right\rangle+\langle\mathcal{S} \boldsymbol{f}, \mathcal{S} \boldsymbol{f}\rangle \\
& =\left\|\left(\mathcal{S}_{h}-\mathcal{S}\right) \boldsymbol{f}\right\|_{0, \Gamma}^{2} \geqslant 0 .
\end{aligned}
$$

Similarly,

$$
\begin{aligned}
\langle\mathcal{S} \boldsymbol{f}, \boldsymbol{f}\rangle-\left\langle\widetilde{\mathcal{S}}_{h} \boldsymbol{f}, \boldsymbol{f}\right\rangle & =\langle\mathcal{S} \boldsymbol{f}, \boldsymbol{f}\rangle-\left\langle\widetilde{\mathcal{S}}_{h} \boldsymbol{f}, \boldsymbol{f}\right\rangle-\left\langle\boldsymbol{f}, \widetilde{\mathcal{S}}_{h} \boldsymbol{f}\right\rangle+\left\langle\widetilde{\mathcal{S}}_{h} \boldsymbol{f}, \widetilde{\mathcal{S}}_{h} \boldsymbol{f}\right\rangle \\
& =\langle\mathcal{S} \boldsymbol{f}, \mathcal{S} \boldsymbol{f}\rangle-\left\langle\widetilde{\mathcal{S}}_{h} \boldsymbol{f}, \mathcal{S} \boldsymbol{f}\right\rangle-\left\langle\mathcal{S} \boldsymbol{f}, \widetilde{\mathcal{S}}_{h} \boldsymbol{f}\right\rangle+\left\langle\widetilde{\mathcal{S}}_{h} \boldsymbol{f}, \widetilde{\mathcal{S}}_{h} \boldsymbol{f}\right\rangle \\
& =\left\|\left(\mathcal{S}-\widetilde{\mathcal{S}}_{h}\right) \boldsymbol{f}\right\|_{0, \Gamma}^{2} \geqslant 0 .
\end{aligned}
$$

Then the min-max property implies that $\lambda_{h} \geqslant \lambda_{h}^{0} \geqslant \widetilde{\lambda}_{h}$.

Since $V_{h}$ is a subset of $\boldsymbol{H}(\operatorname{curl} ; \Omega)$, one has $\lambda \geqslant \lambda_{h}^{0}$. As a consequence, $\widetilde{\lambda}_{h}$ converges to $\lambda$ from below, as concluded in the following proposition.

Proposition 5.2. $\lambda \geqslant \widetilde{\lambda}_{h}$.

Next we consider a refinement $\tau_{h_{i+1}}$ of the mesh $\tau_{h_{i}}$. Thus the finite element spaces for $\tau_{h_{i}}$ and $\tau_{h_{i+1}}$ satisfy $V_{h_{i}} \subset V_{h_{i+1}}$ and $U_{h_{i}}^{\Gamma} \subset U_{h_{i+1}}^{\Gamma}$. Define an eigenvalue problem of finding $\left(\widetilde{\lambda}_{h_{i}}^{h_{i+1}}, \boldsymbol{u}_{h_{i}}\right) \in \mathbb{R} \times V_{h_{i}}$ such that

$$
a\left(\boldsymbol{u}_{h_{i}}, \boldsymbol{v}_{h_{i}}\right)=-\widetilde{\lambda}_{h_{i}}^{h_{i+1}}\left\langle\widetilde{\mathcal{S}}_{h_{i+1}} \boldsymbol{u}_{h_{i}, T}, \boldsymbol{v}_{h_{i}, T}\right\rangle, \quad \forall \boldsymbol{v}_{h_{i}} \in V_{h_{i}} .
$$

Then the $j$-th eigenvalue $\widetilde{\lambda}_{h_{i}, j}^{h_{i+1}}$ is given by

$$
\widetilde{\lambda}_{h_{i}, j}^{h_{i+1}}=-\min _{E_{h_{i}} \in V_{h_{i}}^{(j)}} \max _{\boldsymbol{u}_{h_{i}} \in E_{h_{i}}} a\left(\boldsymbol{u}_{h_{i}}, \boldsymbol{u}_{h_{i}}\right) /\left\langle\widetilde{\mathcal{S}}_{h_{i+1}} \boldsymbol{u}_{h_{i}, T}, \boldsymbol{u}_{h_{i}, T}\right\rangle .
$$

Given $\boldsymbol{f} \in \boldsymbol{L}_{t}^{2}(\Gamma)$, there exists $q_{h_{i}} \in U_{h_{i}}^{\Gamma} \subset U_{h_{i+1}}^{\Gamma}$ such that $\widetilde{\mathcal{S}}_{h_{i}} \boldsymbol{f}=\operatorname{curl}_{\Gamma} q_{h_{i}}$. Hence

$$
\left\langle\widetilde{\mathcal{S}}_{h_{i+1}} \boldsymbol{f}, \widetilde{\mathcal{S}}_{h_{i}} \boldsymbol{f}\right\rangle=\left\langle\boldsymbol{f}, \widetilde{\mathcal{S}}_{h_{i}} \boldsymbol{f}\right\rangle .
$$

Consequently, the same argument as in Lemma 5.1 yields $\left\langle\widetilde{\mathcal{S}}_{h_{i+1}} \boldsymbol{f}, \boldsymbol{f}\right\rangle \geqslant\left\langle\widetilde{\mathcal{S}}_{h_{i}} \boldsymbol{f}, \boldsymbol{f}\right\rangle$, which, by the min-max property, implies that $\tilde{\lambda}_{h_{i}}^{h_{i+1}} \geqslant \widetilde{\lambda}_{h_{i}}$. Using again the min-max property and the inclusion $V_{h_{i}} \subset V_{h_{i+1}}$, we obtain $\widetilde{\lambda}_{h_{i+1}} \geqslant \widetilde{\lambda}_{h_{i}}^{h_{i+1}}$. Hence the following monotonicity of $\widetilde{\lambda}_{h}$ holds.

Proposition 5.3. For meshes $\tau_{h_{i}}$ and $\tau_{h_{i+1}}$ with $\tau_{h_{i+1}}$ a refinement of $\tau_{h_{i}}, \widetilde{\lambda}_{h_{i+1}} \geqslant \widetilde{\lambda}_{h_{i}}$.

\section{Conclusion}

In this work, we propose new definitions of the solution operator $\mathcal{T}$ and two finite element approximations $\widetilde{\mathcal{T}}_{h}$ and $\mathcal{T}_{h}$ for the modified Maxwell's Steklov eigenvalue problem. With the help of these operators, we are able to prove the convergence order of eigenvalues for both finite element methods. Moreover, the ordering between the eigenvalues $\lambda_{h}$ and $\widetilde{\lambda}_{h}$, and the monotonic converging property of $\widetilde{\lambda}_{h}$ are proved. Besides, it is observed in numerical results of [15] that $\lambda_{h}$ has the same monotonic converging property as $\tilde{\lambda}_{h}$. This property of $\lambda_{h}$ is 
interesting to investigate, since, if $\lambda_{h}$ truly converge from below, we can claim using Lemma 5.1 that the finite element method (4.1) is a better choice than (3.1).

Acknowledgements. The research was partially supported by China Postdoctoral Science Foundation Grant 2019M650460.

\section{REFERENCES}

[1] A.B. Andreev and T.D. Todorov, Isoparametric finite-element approximation of a Steklov eigenvalue problem. IMA J. Numer. Anal. 24 (2004) 309-322.

[2] D.N. Arnold, R.S. Falk and R. Winther, Multigrid in H(div) and H(curl). Numer. Math. 85 (2000) $197-217$.

[3] I. Babuška and J.E. Osborn, Eigenvalue problems. In: Handbook of Numerical Analysis, edited by P.G. Ciarlet and J.L. Lions. Vol. II. Elseveier Science Publishers B.V., North-Holland (1991).

[4] S. Bergman and M. Schiffer, Kernel Functions and Elliptic Differential Equations in Mathematical Physics. Academic Press (1953).

[5] D. Boffi, Finite element approximation of eigenvalue problems. Acta Numer. 19 (2010) 1-120.

[6] J.H. Bramble and J. Osborn, Approximation of Steklov eigenvalues of non-selfadjoint second order elliptic operators. In: The Mathematical Foundations of the Finite Element Method with Applications to Partial Differential Equations. Elsevier (1972) 387-408.

[7] A. Buffa and P. Ciarlet Jr, On traces for functional spaces related to Maxwell's equations Part I: an integration by parts formula in Lipschitz polyhedra. Math. Methods Appl. Sci. 24 (2001) 9-30.

[8] A. Buffa, M. Costabel and C. Schwab, Boundary element methods for Maxwell's equations on non-smooth domains. Numer. Math. 92 (2002) 679-710.

[9] A. Buffa, M. Costabel and D. Sheen, On traces for $H(\operatorname{curl}, \Omega)$ in Lipschitz domains. J. Math. Anal. App. 276 (2002) 845-867.

[10] F. Cakoni, D. Colton, S. Meng and P. Monk, Stekloff eigenvalues in inverse scattering. SIAM J. Appl. Math. 76 (2016) 1737-1763.

[11] J. Camaño, C. Lackner and P. Monk, Electromagnetic Stekloff eigenvalues in inverse scattering. SIAM J. Math. Anal. 49 (2017) 4376-4401.

[12] S. Cogar, D. Colton and P. Monk, Using eigenvalues to detect anomalies in the exterior of a cavity. Inverse Prob. 34 (2018) 085006 .

[13] C. Conca, M. Vanninathan and J. Planchard, Fluids and Periodic Structures. Wiley (1995).

[14] M. Costabel, A remark on the regularity of solutions of Maxwell's equations on Lipschitz domains. Math. Methods Appl. Sci. 12 (1990) 365-368.

[15] B. Gong, J. Sun and X. Wu, Finite element approximation of the modified Maxwell's Stekloff eigenvalues, SIAM J. Numer. Anal. 59 (2021) 2430-2448.

[16] M. Halla, Electromagnetic steklov eigenvalues: approximation analysis. ESAIM: M2AN 55 (2021) 57-76.

[17] I. Harris, F. Cakoni and J. Sun, Transmission eigenvalues and non-destructive testing of anisotropic magnetic materials with voids. Inverse Prob. 30 (2014) 035016.

[18] R. Hiptmair and C. Schwab, Natural boundary element methods for the electric field integral equation on polyhedra. SIAM J. Numer. Anal. 40 (2002) 66-86.

[19] Q. Li, Q. Lin and H. Xie, Nonconforming finite element approximations of the Steklov eigenvalue problem and its lower bound approximations. App. Math. 58 (2013) 129-151.

[20] J. Liu, J. Sun and T. Turner, Spectral indicator method for a non-selfadjoint Steklov eigenvalue problem. J. Sci. Comput. 79 (2019) 1814-1831.

[21] P. Monk, Finite Element Methods for Maxwell's Equations. Oxford University Press (2003).

[22] D. Mora, G. Rivera and R. Rodríguez, A virtual element method for the Steklov eigenvalue problem. Math. Models Methods Appl. Sci. 25 (2015) 1421-1445.

[23] J. Sun and A. Zhou, Finite Element Methods for Eigenvalue Problems. CRC Press, Boca Raton, London, New York (2016).

[24] H. Xie, A type of multilevel method for the Steklov eigenvalue problem. IMA J. Numer. Anal. 34 (2014) 592-608.

[25] F. Xu, M. Yue, Q. Huang and H. Ma, An asymptotically exact a posteriori error estimator for non-selfadjoint Steklov eigenvalue problem. Appl. Numer. Math. 156 (2020) 210-227.

[26] Y. Yang, Q. Li and S. Li, Nonconforming finite element approximations of the Steklov eigenvalue problem. Appl. Numer. Math. 59 (2009) 2388-2401. 
[27] Y. Yang, Y. Zhang and H. Bi, Non-conforming Crouzeix-Raviart element approximation for Stekloff eigenvalues in inverse scattering. Adv. Comput. Math. 46 (2020) 1-25.

\section{Subscribe to Open (S20) A fair and sustainable open access model}

This journal is currently published in open access under a Subscribe-to-Open model (S2O). S2O is a transformative model that aims to move subscription journals to open access. Open access is the free, immediate, online availability of research articles combined with the rights to use these articles fully in the digital environment. We are thankful to our subscribers and sponsors for making it possible to publish this journal in open access, free of charge for authors.

\section{Please help to maintain this journal in open access!}

Check that your library subscribes to the journal, or make a personal donation to the S2O programme, by contacting subscribers@edpsciences.org

More information, including a list of sponsors and a financial transparency report, available at: https://www. edpsciences.org/en/maths-s2o-programme 